\title{
El futuro de la Universidad y la Universidad del Futuro. Ecosistemas de formación continua para una sociedad de aprendizaje y enseñanza sostenible y responsable
}

\author{
Emilio Álvarez-Arregui (*) y Xavier Arreguit (**) \\ $\left({ }^{*}\right)$ Universidad de Oviedo (España) \\ ${ }^{* *}$ Innobridge S.L. (Lausanne-Suiza) \\ Emilio Álvarez-Arregui
}

Profesor Titular de la Universidad de Oviedo en el Departamento de Ciencias de la Educación, actualmente es director del Área del Instituto de Investigación e Innovación Educativa. Ha sido investigador principal en proyectos autonómicos, nacionales e internacionales. En este momento focaliza su atención en el desarrollo de modelos de desarrollo de competencias con universidades, institutos de investigación, compañías, administraciones, consejerías, agentes sociales y plataformas de aceleración de la innovación de diferentes países americanos y europeos. Tiene más de dos centenares de publicaciones en revistas y libros relacionadas con estas y otras temáticas. Ha realizado estancias docentes e investigadoras en universidades españolas (Sevilla, Huelva, León, Islas Baleares, Jaén, UNED...) y extranjeras (Uruguay, Chile, México, Letonia, Portugal, EEUU, Reino Unido, Irlanda, Brasil, Suiza). Es miembro de la Sociedad Española de Pedagogía, del Grupo "Universidades y Educación Inclusiva", del "Servicio Español para la Internacionalización de la Educación (SEPIE)", de la "Red Europea de Educación Inclusiva y Discapacidad incluDed"; del "Fórum Europeo de Administradores de la Educación (FEAE)"; de la Red Transdisciplinar de Investigación Educativa (RETINDE); de la Red de Universidad Inclusivas (RUIE); de la Red de Institutos de Investigación Educativa de España (RIIE); de la Red Euroamericana de Educomunicación Alfamed; de la Red Iberoamericana de Evaluación de la Calidad Educativa de la Educación Superior (RIAICES); de la Red Universitaria de Estudios de Posgrado y Educación Permanente (RUEPEP); y de la Red UNESCO en el Principado de Asturias, entre otras. Es director de la Revista Aula Abierta.

ORCID: https://orcid.org/0000-0002-4657-753X alvarezemilio@uniovi.es

\section{Xavier Arreguit}

Managing Director and President of Innobridge SA and Innobridge Services Sàrl. He holds a MS Degree in Engineering and a PhD from the Swiss Federal Institute of Technology EPFL, and he subsequently obtained an Executive MBA at HEC-Lausanne / EPFL. After a postdoc in the Computer and Neural Sciences Department at the California Institute of Technology (CALTECH) in United States, he worked at the CSEM in Neuchâtel (where he was vice-president, in charge of Strategic Marketing and Business Development). In 2004, he co-founded Innobridge SA, now a holding company, and contributed in 2005 to the creation and management of JADE Invest SA, a subsidiary of CSEM, specialized in investing in start-ups. In 2010, he co-founded Innobridge Services Sàrl, a strategy and management consulting company with innovation expertise in Smart Cities, Industry 4.0, Cleantech, Sport, Medtech and Education. He has also contributed to several patents and products and to the creation, financing, development and sale of several start-ups. He has developed expertise in systems and innovation models involving public-private partnerships and has participated as an invited external lecturer in several master courses. He is a member of several Companies' Boards of Directors in Switzerland and in Malaysia.

\section{RESUMEN}

La Sociedad actual se caracteriza por su carácter complejo, interdependiente, dinámico e informacional en la que concurren personas físicas y personas jurídicas que se integran en un medio ambiente político, social, económico, ecológico, físico-finito y con recursos limitados. En este contexto se van consolidando temporalmente y de manera interdependiente distintas macrotendencias que aceleran los procesos de manera exponencial en todos los ámbitos lo que hace germinar nuevos modelos económicos, políticos y sociales que obligan a las personas a una adaptación permanente de sus competencias, necesarias para evitar que se generen polarizaciones, exclusiones y brechas de alfabetización insalvables.

Las universidades que han sido lugares de generación y de transmisión de conocimiento deben entender, aceptar y adaptarse al contexto emergente y anticipar futuras situaciones ya que de no hacerlo sus misiones quedarán abocadas a la irrelevancia y serán desplazadas como agentes y referentes educativos. En este artículo se hace un recorrido por todas estas cuestiones y se plantea un modelo ecosistémico para la formación contextualizado a las nuevas exigencias del Espacio Europeo de Educación Superior y tiene en cuenta los principios que guían la Agenda 2030 de Desarrollo Sostenible. Estos referentes se están materializando en el Instituto de Investigación e Innovación Educativa (INIE) de la Universidad de Oviedo a través de proyectos de investigación e innovación educativa y de programas transversales de formación.

Palabras Clave: aprendizaje, enseñanza, formación, ecosistema, cambio e innovación educativa. 


\section{Contextualización}

La íntima conexión que se ha establecido entre Medio Ambiente, Sociedad, Economía, Legislación, Conocimiento, Tecnología, Trabajo, Ocio y Educación en el entorno vital de las personas físicas, individuos, y jurídicas, organizaciones, hace necesario que los responsables públicos y privados estén teniendo que desarrollar nuevas visiones, misiones y estrategias glocales para establecer ajustes ante los desequilibrios que emergen en el sistema y los subsistemas como consecuencia de la concurrencia de las macrotendencias marco y las variables situacionales concurrentes.

A día de hoy, es una realidad palpable que el desarrollo científico y tecnológico proporciona beneficios para una gran parte de la población en términos absolutos. Castells (1999, 2000) ya nos indicaba hace dos décadas que el acceso a los recursos, bienes y servicios era desigual por selectivo. Esta situación la asociaba a la búsqueda constante de la rentabilidad sobre el desarrollo social y medioambiental sostenible. Reflejo de ello son las asimetrías que nos encontramos en el crecimiento entre las regiones, los países, las ciudades o las rentas vitales de las personas. En este proceso se han ido generando movimientos centrífugos y centrípetos que van dando lugar a agujeros negros en el mundo y en las ciudades que han provocado conexiones perversas que no siempre son visibles pero si deben ser denunciadas. En estas zonas desfavorecidas aparece de manera recurrente una relación con una gestión deficiente de lo público que se manifiesta con redes de servicios endebles o en continuo deterioro con la pobreza, el analfabetismo funcional o la explotación que llevan aparejados. Estos entornos son el mejor caldo de cultivo para que se consoliden círculos viciosos que incapacitan a las personas físicas para incorporarse, mantenerse y desarrollarse profesionalmente en sus entornos sociolaborales $\mathrm{y}$ a las personas jurídicas a generar un tejido productivo responsable social y medioambientalmente o desplegar redes que proporcionen soportes vitales básicos a la ciudadanía en sus regiones y comunidades locales.

En el ámbito educativo Toffler $(1990 ; 1991)$ visualizó a finales del siglo pasado que el desarrollo tecnológico tendría múltiples consecuencias. A este respecto nos anunció que nos iba a proporcionar grandes oportunidades por la interactividad (capacidad bidimensional de respuesta, alumno-máquina y viceversa), la movilidad (capacidad de desarrollar educación en cualquier ecosistema lo que romperá con el monopolio de las instituciones educativas clásicas, la Escuela y la Universidad), la convertibilidad (capacidad de transmitir y procesar información entre medios y redes diferentes a fin de conformar sistemas complejos y multivariadas de uso común), la conectabilidad (conexión entre los agentes educativos con fuentes plurales de información), la omnipresencia (democratización total de la información) y la mundialización (información sin fronteras ni diferencias). El cambio de modelo vendría acompañado de brechas de acceso y alfabetización de distinto signo por la aparición de nuevas variables que tendrían como característica común su continua mutación.

Entre otras predicciones que se vienen haciendo realidad queremos referimos al hecho de cómo la dispersión y la descentralización de la educación nos va acercando a la necesidad de adoptar una concepción adhocrática de lo organizativo cuando los responsables de lo educativo priman en los sistemas un incremento de las tareas burocráticas, el individualismo, la especialización, la estandarización y la competitividad ya que desde estos referentes culturales se está limitando no solo la capacidad de creatividad a las personas sino la de desarrollar, aplicar, y transferir conocimiento. Este enfoque no solo está reduciendo la capacidad de las instituciones para cubrir sus misiones sino que desprofesionaliza a los responsables directos de la educación. Así, muchas tareas que se les encomiendan no son propias de su función y han colonizado su tiempo de trabajo lo que ha ido en detrimento de una docencia, investigación e innovación educativa de calidad.

Las argumentaciones a favor y en contra de la situación generada se solapan. En los foros especializados se reconoce, explícita e implícitamente, las limitaciones de los sistemas actuales para abordar el futuro de una Sociedad donde el Aprendizaje y la Enseñanza continua serán el referente para seguir en todos los ámbitos de nuestras vidas. En cualquier caso, se quiera o no, las transformaciones tendrán que afrontarse irremediablemente y se tendrá que asumir que son de hondo calado ya que no solo afectarán a las estructuras, la gestión, las funciones, los espacios, los tiempos y los recursos sino que se también están implicadas las actitudes y los valores que impregnan las culturas organizativas. Por tanto, en cualquier propuesta de mejora, el saber, el saber hacer y el saber ser tendrán que estar íntimamente conectadas.

Las reformas educativas que se están implementando en diferentes contextos, y cada vez en períodos más cortos de tiempo, son las herramientas que utilizan los políticos para favorecer la adaptación al cambio. El problema que está emergiendo es que, a pesar de la legitimidad del procedimiento, las normas no parecen estar orientadas desde los principios que guían un desarrollo sostenible para las personas físicas y jurídicas, en general, y en el entorno universitario, en particular. La obsesión por la competitividad intra e interinstitucional, el valor que se concede a los rankings o los protocolos que se imponen por las agencias evaluadoras externas se generalizan a través de las memorias de verificación. Las Facultades, las Escuelas, los Centros y los Institutos de Investigación se han visto obligadas a estandarizar los procesos detallando en los currículums los contenidos, las competencias, las metodologías, los tiempos, los espacios, los recursos, las relaciones o los sistemas de evaluación, entre otras cuestiones, pero no se cuestionan los procedimientos seguidos o los resultados colaterales que se han integrado en las culturas organizativas. En nuestra opinión este planteamiento ha venido agotando al sistema y lo está poniendo al borde de la paralización porque lo incapacita para desarrollarse y dar respuestas autónomas y flexibles adaptadas a las necesidades, expectativas y demandas que se generan situacionalmente, por lo que parecen necesario volver a recordar algunas cuestiones que se van obviando por sobreentendidas, obviadas $u$ oxidadas pero que siguen vigentes (Álvarez-Arregui, 2017).

A este respecto debe indicarse que muchas personas físicas y jurídicas responsables de lo educativo parecen haberse olvidado en la práctica de que su responsabilidad académica, social y laboral les exige dotar a los agentes implicados de las herramientas necesarias para preparar a las nuevas cohortes de profesionales y de estudiantes que entran, transitan, egresan y reingresan en el sistema para incorporarse a una economía del conocimiento, del aprendizaje y de la enseñanza continuada, como ciudadanos responsables que fortalecen la sociedad civil.

Los administradores de las instituciones educativas públicas también parecen estar olvidando que la innovación es un proceso continuado que requiere creatividad para dar respuesta a las situaciones problemáticas que emergen desde el desarrollo de proyectos multidisciplinares fundamentados en el conocimiento donde las personas físicas y jurídicas desarrollen ideas, herramientas, modelos, protocolos... en definitiva innovaciones que deben integrarse en las culturas curriculares académicas para que las universidades los implementen, los desarrollen y los mejoren en sus grados, másteres, títulos propios y programas transversales de formación 
continua. Estas cuestiones cuando dejan de ser objeto de atención preferente o de debate dentro y fuera de los sistemas universitarios hacen que se orienten los procesos, las personas y las instituciones hacia una estandarización mecánica donde las regulaciones, las rutinas, la competitividad y la burocratización se acaban convertido en la norma en lugar de la excepción (Álvarez-Arregui, 2018, 2019).

Los administradores de lo educativo también parecen haber olvidado que en los próximos años, en nuestro entorno cultural, una amplia cohorte de profesionales, incluidos los docentes, que empezaron a trabajar en la época de expansión (1970-1985) se están jubilando y serán parcialmente reemplazados. Esta situación podría derivar a medio y largo plazo en un progresivo deterioro de muchas instituciones universitarias, pero sobre todo de aquellas que no hayan previsto, reflexionado y actuado sobre los sistemas de selección actuales donde se ha instalado una fuerte endogamia y un clientelismo que mantiene, cuando no incrementa, la precariedad de los servicios públicos. Actualmente sigue sin quererse intervenir sobre una cultura organizativa que en la educación superior se ha politizado en exceso y gira alrededor de grupos celulares independientes o con baja relación funcional donde los sistemas de coordinación intermedia se han burocratizado y donde los equipos docentes, investigadores y administrativos pueden diseñar, innovar, aplicar o experimentar la excelencia o el fracaso independientemente del resto de unidades o de la institución que las acoge (Buckland, 2009). La ambigüedad de objetivos, la diversidad de intereses y las luchas por el poder (Weick, 1967) se perpetúan en muchas instituciones universitarias ralentizando cuando no imposibilitando el desarrollo de visiones, misiones y estrategias con proyección de futuro, lo que favorece la competición y el enfrentamiento en detrimento de la colaboración, de la formación continua y de la mejora sostenible.

Los administradores de lo educativo parecen haber olvidado que la sociedad postmoderna ya no es susceptible de incorporar verdades absolutas e inamovibles como ocurría en la modernidad. Hoy en día la persona se vuelve a resituar en el centro del proceso y, al igual que Colom (1997), nos parece necesario reorientar la misión de la educación hacia el saber donde se reinterprete el humanismo avalando un hombre autosuficiente. A este respecto nos parecen apropiadas las aportaciones de $\underline{\text { Toffler }}$ $(1990,1991)$ cuando manifestaba que educar sería aprender a innovar, aprender a entender el cambio, aprender a cambiar y aprender a vivir con el cambio. Si se asumen como válidas sus argumentaciones habrá que desplegar estrategias en la reinterpretación de la docencia y de los docentes porque al situar el aprendizaje como foco de atención prioritario en la sociedad del futuro, la responsabilidad de la educación ya no podrá estar sólo en manos de los maestros y profesores, por lo que habrá que tomar decisiones conjuntas desde las culturas políticas, académicas, sociales y empresariales. El currículum deberá ser, por tanto, objeto de profundas revisiones para ir dotándolo de una base común donde la innovación sistemática, sistémica y continua requerirá una formación a lo largo de la vida, lo que avala el desarrollo, despliegue y consolidación de un currículum con una base universal de aprendizaje y enseñanza (Álvarez-Arregui, Rodríguez-Martín y otros, 2015); por último, se hace necesario revisar con detenimiento los resultados que se obtienen desde la investigación educativa fundamentada y contrastada para incorporarlos de una manera circular en el sistema a través de múltiples combinaciones.

En el fondo, estos cambios que anunciamos como necesarios son reacciones lógicas de las personas físicas y jurídicas para adaptarse a una sociedad en constante evolución. Como suele ser habitual ha sido la gran empresa la que ha explorado en primer lugar las posibilidades que ofrece el nuevo escenario socioeducativo y laboral, indicando que aquellas organizaciones que quieran asegurar su continuidad y existencia deben promover sistemas de formación permanente para sus trabajadores, donde la creatividad, el emprendimiento, el trabajo en equipos multidisciplinares, el pensamiento crítico, la resiliencia, la actitud positiva ante el error o la responsabilidad social sean el corpus de referencia del curriculum para el desarrollo de las competencias genéricas y específicas. En nuestro caso, consideramos que la intervención en este complejo escenario demanda la construcción de visiones ecosistémicas dinámicas sostenibles que integren las culturas políticas, académicas, empresariales y sociales a través de múltiples relaciones que se combinarán de arriba-abajo, de abajo-arriba, lateralmente y de manera transversal.

Somos conscientes de que la construcción de ecosistemas de formación sostenibles que conjuguen una formación inclusiva en competencias genéricas y específicas será difícil porque la enseñanza, el aprendizaje y el curriculum ya no son patrimonio de ninguna institución en concreto, pero será posible si adoptamos una perspectiva sistémica en la que participen de manera responsable y comprometida todos los agentes con responsabilidad en lo educativo. Por tanto, nos parece necesario presentar algunas lecciones que hemos aprendido y enseñado en las últimas décadas sobre las posibilidades y dificultades que acompañan al cambio en las organizaciones, ya que bajo este planteamiento podremos contextualizar la evolución que se ha seguido en el desarrollo del Espacio Europeo de Educación Superior y nos permitirá cerrar el artículo con una propuesta de futuro en la que estamos trabajando actualmente.

\section{El cambio cultural: Lecciones aprendidas y enseñadas desde la experiencia}

Actualmente atravesamos un momento que cabría calificar como crítico ya que son muchos los nuevos escenarios que emergen en la sociedad, lo que exige a las personas físicas y jurídicas a buscar respuestas para adaptarse continuamente en todos los ámbitos en los que se desenvuelven de ahí que la I+D+i esté pasando a formar parte inherente de nuestros entornos personales, profesionales y sociales.

El problema del cambio es su polisemia y se ha ido asociando a otros conceptos como mejora, desarrollo, reforma o renovación, pero también con ruptura (Popkewitz, 1994) con lo estable o con lo natural de nuestra vida social. Por tanto, cuando se habla sobre el cambio, debe tenerse presente que son muchas las perspectivas que pueden adoptarse para abordarlo y no siempre se toman las decisiones más acertadas. En nuestro caso y en este contexto, nos ha parecido oportuno presentar algunas lecciones que hemos aprendido y enseñado desde el ámbito académico, social y empresarial donde somos testigos que la I+D+i Educativa actúa a manera de savia, de energía interna, que circula por el sistema público y privado. Algunos caminos ya se han trillado, por lo que haremos un breve recorrido por algunos de los que hemos transitado, ya que consideramos que pueden servir de orientación, guía o referencia para desarrollar visiones, misiones y estrategias desde las que se exploren nuevas vías para lograr una implementación real y generalizada de la I+D+i Educativa.

\section{Lección I}

La investigación y la práctica coinciden en que el cambio educativo ocurre se quiera o no, dado que las organizaciones 
educativas, entendidas como ecosistemas de formación intencionales, están en continua transformación, al igual que la sociedad de la que forman parte, como efecto de la globalización; del desarrollo científico-tecnológico; del neoliberalismo; y del acceso a los datos, la información, el conocimiento y el saber. Esta situación supone un cambio de paradigma porque requiere adquirir o desarrollar nuestras competencias a través del aprendizaje, la enseñanza y el esfuerzo personal ya que las decisiones que se adopten a este respecto tendrán efectos sobre los ejes que orientan la vida de las personas (social; laboral; cultural-ocio; personal; y político) con repercusiones de distinto signo (Álvarez-Arregui, 2010; Álvarez-Arregui, Rodríguez-Martín, Agudo Prado y Arreguit, 2017; Álvarez-Arregui, Rodríguez-Martín; Madrigal Maldonado, Grossi -Sampedro y Arreguit, 2017).

\section{Lección II}

Las plataformas conceptuales, los modelos y las reformas que se desarrollan desde la política van a remolque de la práctica, dado que el cambio se conoce una vez que se ha producido y la innovación ha sido asumida por las personas usuarias. Por tanto, las visiones fundamentadas exclusivamente en los resultados se han mostrado como insuficientes, de ahí que deba ampliarse el foco hacia visiones sistémicas donde deberán tenerse en cuanta los factores de entrada, de proceso y de producto desde diferentes dimensiones, momentos, escenarios y perspectivas (Álvarez-Arregui, 2002; Arreguit, 2017).

\section{Lección III}

El cambio en los ecosistemas socioeducativos afecta al entorno, la población, la tecnología y los sistemas de relaciones, por tanto, modifica o influyen en las personas, los procesos, los programas, los resultados, el clima y la cultura. En el plano personal y profesional se produce el cambio cuando se construye una visión sistémica sobre lo educativo que tiene en cuenta las problemáticas que lo acompañan, las posibilidades que proporciona la colaboración, el valor de la investigación para generar conocimiento, ponerlo en funcionamiento y mejorar las competencias y el incremento de la calidad de vida de las personas usuarias a través del aprendizaje y la enseñanza de unos valores compartidos (Álvarez-Arregui, 2017).

\section{Lección IV}

En el plano organizativo se favorece el cambio cuando se construye una visión de conjunto compartida y se generan estructuras, normas y prácticas coherentes con esa visión, capacidad de generar desarrollo profesional, institucional, comunitario y conocimiento que haga crecer a las personas, a los equipos y a la institución en una cultura de colaboración en la que se comparten los avances y se proporcionan apoyos (internos y externos) ante las dificultades (Álvarez-Arregui, 2002).

\section{Lección $V$}

El cambio genera incertidumbre porque las macrotendencias generan tensiones, caos e inestabilidad en los ecosistemas socioeducativos lo que requiere tomar decisiones colegiadas sobre la organización, el curriculum y el desarrollo profesional. La incertidumbre se reduce cuando el cambio se justifica públicamente y de manera fundamentada (por qué, para qué, qué se pretende, a quién afecta, quién lo promueve...) a las diferentes audiencias en función del momento político, económico, social y cultural, se les hace partícipes en la construcción de la visión, la misión, la estrategia y la implementación y participan del impacto (Álvarez-Arregui, 2009; Álvarez-Arregui, Rodríguez-Martín, Agudo Prado y Arreguit, 2017).

\section{Lección VI}

Los cambios en los ecosistemas socioeducativos deben ser impulsados simultáneamente de arriba-abajo (reformas), de abajo arriba (innovación y desarrollo organizativo-comunitario), lateralmente (profesionalización, coordinación y colaboración interinstitucional) y transversalmente (proyectos y equipos pluridisciplinares) con la intención de aprender a aprender, aprender a cambiar, aprender a aceptar el cambio, aprender a gestionar el cambio y aprender a vivir con el cambio (Álvarez-Arregui, Rodríguez-Martín; Madrigal Maldonado, Grossi -Sampedro y Arreguit, 2017).

\section{Lección VII}

La colaboración se convierte en un referente clave para abordar el cambio y la incertidumbre cuando se interpreta como: a) apoyo moral para hacer emerger los aspectos vulnerables ayudando a afrontar los fracasos y las frustraciones que conlleva este proceso; b) aumento de la eficiencia, dado que la coordinación elimina las duplicidades y las redundancias entre los contenidos que se imparten; c) mayor eficacia, puesto que se mejora la calidad del aprendizaje al mejorar la calidad de la enseñanza del profesorado; d) apoyo mutuo para afrontar riesgos, incorporar una mayor diversidad de estrategias, incrementar la retroalimentación y generar un clima de confianza; e) reducción del exceso de trabajo burocrático al compartirse las cargas y las presiones; f) visiones temporales sincronizadas al participar de una misma perspectiva sobre la orientación del cambio a partir de la situación existente ya que le confiere mayor credibilidad; g) certeza situacional asentada en las posibilidades reales de actuación y las limitaciones existentes; h) asertividad política, ya que el trabajo en equipo refuerza la confianza para adoptar propuestas externas y se espera el momento más apropiado para plantear su implementación, retrasarla u oponerse en el caso de que resulte disfuncional; i) incremento de la capacidad de reflexión como consecuencia del trabajo conjunto, de la comunicación y de las acciones conjuntas; j) mejora de la capacidad de respuesta de la organización, ya que son procesos sinérgicos producto de las capacidades y conocimientos que se ponen en común; k) incremento de las oportunidades para aprender y enseñar fruto del intercambio y de la actitud positiva en el aula, en el departamento, en la facultad, con las familias, con el alumnado, con otros ecosistemas educativos...; 1) perfeccionamiento continuo al entender el cambio como un proceso que no tiene fin en la búsqueda de nuevas soluciones a las situaciones problemáticas que se presenten (Hargreaves, 1998; Álvarez-Arregui; 2017; Arreguit, 2017).

\section{Lección VIII}

El cambio y la colaboración son objeto de múltiples interpretaciones dado que hay muchos intereses e interesados a su alrededor lo que genera desviaciones de los ecosistemas educativos hacia la complacencia, si se orientan hacia lo seguro y se evita el conflicto; el conformismo, si degenera en una cosificación del grupo que elimina la creatividad individual; la artificialidad, si se monopolizan los procesos desde la administración y se tratan superficialmente; y la cooptatividad, cuando se utiliza políticamente la colaboración para asegurarse la condescendencia del 
profesorado con las reformas educativas. Por tanto, el cambio y la colaboración pueden adoptar diferentes vías en función de quién lo controla, quién participa, cuáles son sus fines y qué condiciones deben establecerse para mantenerlo. Las desviaciones siempre están latentes porque son intrínsecas a cualquier proceso de adaptación de ahí que tendrán que articularse propuestas de intervención situacionales asentadas en unos comportamientos éticos compartidos por las personas concurrentes y donde la dirección y el estilo de liderazgo jugarán un papel fundamental en su promoción, defensa, desarrollo, implementación, revisión y sostenibilidad (Hargreaves, 1989; Álvarez-Arregui, Rodríguez-Martín, Agudo Prado y Arreguit, 2017).

\section{Lección IX}

Las causas que dificultan el aprendizaje, la enseñanza y el emprendizaje en las organizaciones se deben a múltiples factores. En unos casos son endógenas, en otras exógenas y en las mayorías de los casos mixtas y vinculadas a las culturas organizativas que se hayan ido consolidando. La última parece la más adecuada dada la interdependencia que se genera entre unas instituciones socioeducativas que se desarrollan situacional y coyunturalmente y unas demandas que se generan en una sociedad globalizada, con un alto desarrollo tecnológico, con una capacidad de acceso a los datos, la información y el conocimiento ilimitada, con unas redes sociales que impregnan todos los ámbitos de nuestras vidas y con un neoliberalismo hegemónico que condiciona las políticas sociales y educativas y, por tanto, los proyectos en las instituciones (Álvarez-Arregui, 2002; Arreguit, 2018).

\section{Lección X}

El cambio requiere facilitadores: la motivación (disponibilidad, ambiente de interacción, refuerzo del trabajo en equipo, animación del dialogo...); la justificación (recordando el propósito del cambio y reforzando periódicamente el compromiso relacionando el proceso con las tareas habituales...); el apoyo político (localizar a aquellos agentes externos-internos que pueden bloquear o distorsionar el proceso de cambio estableciendo contactos para mejorar las relaciones, bloquear las resistencias, buscar su conformidad...); y la sostenibilidad (gestionar el cambio aportando recursos, incentivos, formación, asesoramiento, visibilización...). Además, deben tomarse decisiones sobre los dilemas que emergen en el proceso (individual, de equipo, departamental, institucional, comunitario, estructural, organizativo...) (Álvarez-Arregui, 2017).

\section{Lección XI}

El cambio viene acompañado de estrategias de distinto signo: coercitivas (determinadas desde la administración), racionales (confían en que su diseño conllevará una aceptación, interiorización y puesta en marcha) o reeducativas (orientadas a lograr el compromiso de las personas concurrentes para que avalen la mejora adecuando el contexto y las formas de pensar y de hacer). Pueden aplicarse en las diferentes fases del cambio: inicio (sensibilización, difusión y debate del conocimiento); desarrollo (revisión basada en la escuela); e institucionalización (evaluación del proceso de cambio) (Bolivar, 1999; Álvarez-Arregui, 2017).

\section{Lección XII}

El cambio debe ser sostenible, de ahí que haya que tener en cuenta una serie de claves asociadas a la mejora si se quiere ir más allá de los procesos de escolarización. Así, debe perdurar, debe contar con suficientes recursos disponibles o alcanzables, no debe perjudicar a otras instituciones y sistemas y debe promover la diversidad y la capacidad ecológica en todo el entorno educativo y comunitario (Fullan, 1991; Álvarez-Arregui, Rodríguez-Martín; Madrigal Maldonado, Grossi-Sampedro y Arreguit, 2017).

\section{Lección XIII}

La sostenibilidad se fortalece cuando se integran las diferentes culturas locales en el proceso reformista de modo que puedan ser reinterpretadas situacionalmente desde un diálogo constructivo que favorezca la diversidad, algo que va en contra de las actuales reformas que se están promoviendo a nivel internacional, donde la comparación estandarizada al imponerse como norma incrementa la desigualdad (Hargreaves y Fink, 2002; Álvarez-Arregui, 2017).

\section{Lección XIII}

El cambio y el liderazgo están asociados, de ahí que aquellas personas que quieran promoverlos con proyectos, que ocupen cargos de responsabilidad por designación, o que las haya elegido la organización para su gestión deben conocer las evidencias empíricas y las buenas prácticas que avalan los procesos de mejora que han sucedido o se desarrollan en otras organizaciones del mismo ámbito. La comunicación, la delegación, el fomento de la innovación y la humildad son referentes básicos en la gestión de las organizaciones y de los ecosistemas I+D+i (Arreguit, 2018).

\section{Lección XIV}

La revisión de estudios empíricos y desde la propia práctica nos indican que no se puede mandar lo que debe hacerse; el cambio es un viaje; los problemas son nuestros amigos; la visión se va construyendo entre todas las personas; el individualismo y el colectivismo tienen igual poder; ni la centralización ni la descentralización funcionan aisladamente; cualquier persona puede convertirse en un agente de cambio; las culturas organizativas internas tamizan las prescripciones externas; las relaciones con el entorno pueden ser críticas; los planteamientos lineales son insuficientes; y la construcción de conocimiento organizativo válido es complejo porque tiene que ser capaz de relacionar, contextualizar y globalizar de manera secuencial y simultánea en distintos planos (Fullan, 1991; Álvarez-Arregui, 2017).

\section{Lección XIV}

Las instituciones socioeducativas han ido mermando progresivamente su capacidad de aprendizaje y enseñanza porque los documentos institucionales que utilizan para orientar su desarrollo no han cristalizado como referentes y guías de las estrategias de intervención. La orientación burocrática que les han imprimido aquellos responsables institucionales que han ejercido unos liderazgos transaccionales, ha alentado, explícita o implícitamente, unos criterios estandarizados que han venido respaldando aquellas administraciones educativas que prefieren el control y la dependencia al desarrollo y la mejora continua o que estas organizaciones se gestionen bajo criterios de mayor autonomía y de participación de la comunidad educativa (Álvarez-Arregui, 2002). 


\section{Lección XVI}

No se percibe que haya existido una intencionalidad de mejora educativa real por parte de muchos promotores institucionales, externos e internos, de apoyarse en documentos institucionales que recojan visiones, misiones y estrategias compartidas para proyectar a sus comunidades de referencia hacia la construcción de una cultura de colaboración inter e institucional fuerte donde se reflejasen los intereses, demandas, necesidades y expectativas de los diferentes sectores. Por tanto, no se ha producido una identificación con los principios que los guían y se han acabado generalizado como documentos administrativos (Álvarez-Arregui, 2007).

\section{Lección XVII}

La paradoja que nos hemos encontrado en aquellas instituciones con propuestas organizativas más desarrolladas asentadas en planteamientos integrales, con proyectos en marcha en todas las etapas y departamentos, con propuestas de arriba-abajo y de abajo-arriba, con unos niveles de participación altos y con una dirección más pedagógica, transformacional y profesional, es que no consideran que su Proyecto Institucional y/o su Plan Estratégico esté concluido, sino que los interpretan como un proceso dinámico en continuo desarrollo, por lo que utilizan distintos sistemas de retroalimentación para mejorarlos constantemente (Álvarez-Arregui, 2017).

\section{Lección XVIII}

El aprendizaje de las personas jurídicas, las organizaciones, se ha visto afectado negativamente cuando los equipos directivos y los sistemas de coordinación intermedia no han asumido su responsabilidad institucional y social en el desarrollo de proyectos compartidos. En estos casos han preferido optar por modelos organizativos restrictivos donde prevalecen las propuestas jerárquicas sobre las horizontales, donde unos colectivos emergen y otros quedan relegados, donde los órganos unipersonales y colegiados se utilizan como mecanismo de legitimación formal de las políticas institucionales y donde se ha secuestrado la reflexión, el debate constructivo, la planificación de la intervención, el despliegue de los procesos y la toma de decisiones en base a revisiones burocráticas orientadas al mantenimiento (Álvarez-Arregui, 2017; Álvarez-Arregui, Rodríguez-Martín, Agudo Prado y Arreguit, 2017).

\section{Lección XIX}

El aprendizaje y la enseñanza en las organizaciones se resiente cuando la apatía hacia la planificación se incrementa ante la tramitación rutinaria de unos documentos institucionales cuyo principal objetivo es justificarse ante las administraciones educativas. A esta situación no se llega por azar sino por la concurrencia de procesos que se consolidan en el tiempo, amparados en una planificación formal, académica y homogénea que se legitima en los ritos simbólicos institucionales a sabiendas de las incongruencias existentes (Álvarez-Arregui, 2017).

\section{Lección XX}

En las organizaciones que tienen dificultades para aprender y enseñar se han detectado deficiencias comunes en la forma en que las direcciones o los responsables institucionales plantean el cambio. A este respecto debe tenerse presente que cuando se quieren dirigir organizaciones, no deben hacerse propuestas de futuro si no se dan las condiciones necesarias, ya que esta situación se acabará volviendo contra los promotores si se producen disonancias entre lo que se dice que se va a hacer, lo que se hace realmente y lo que se puede hacer (Álvarez-Arregui, Rodríguez-Martín; Madrigal Maldonado, Grossi -Sampedro y Arreguit, 2017).

\section{Lección XXI}

Las personas que quieren asumir una responsabilidad institucional deben ser conscientes que son muchas las actitudes que pueden adoptarse ante las visiones de la organización, de ahí que sea relevante comunicar la información con la mayor objetividad posible, cosa que no suele ocurrir cuando está salpicada de opiniones preconcebidas, prejuicios personales e ideología. Al presentar el futuro deseable para la institución debe tenerse presente que: a) el verdadero compromiso es raro, los directivos pretenden que los trabajadores participen de la visión, pero ese planteamiento se asienta en un liderazgo transaccional b) el alistamiento deja libertad de elección y, aunque se respalda la visión, aún no se siente copartícipe, por lo que habrá que abrir espacios para compartirla, incorporando otras aportaciones y hacerlos partícipes de ella; c) ante la desobediencia y la apatía habrá que intentar escuchar en unos casos las razones y en otros intentar ilusionar, pero habrá que actuar situacionalmente en función de las circunstancias reflexionando sobre los por qués, pero siendo conscientes de que lo mejor es evitar la hipocresía o generar falsas expectativas que a la larga deriven en enfrentamientos, chantajes encubiertos y en conflictos abiertos; y d) cuando se parte o se adoptan posiciones de partida excluyentes, poco se puede hacer (Senge, 1990; Álvarez-Arregui, 2017).

\section{Lección XXII}

El balance de las reformas varía en función del grado de difusión de sus fines, de las estrategias y de la metodología que la fundamenten, pero, sobre todo, deben contar con un amplio apoyo social, promoverse el mayor consenso político posible, plantearse a largo plazo, sensibilizar a los agentes implicados y evitar imposiciones organizativas y curriculares. Estos condicionantes no pueden desvincularse del papel regulador que tienen las administraciones públicas y los organismos nacionales e internacionales. Estableciendo distancias con las políticas curriculares partidistas consideramos necesario apoyar las iniciativas institucionales, velar porque no se promuevan desigualdades educativas y apoyar un sistema educativo público, democrático, transparente, inclusivo, creativo, emprendedor e innovador (Álvarez-Arregui, 2017).

\section{Lección XXIII}

En el proceso de cambio se debe prestar atención al liderazgo institucional, a la actitud del profesorado, a la implicación de la comunidad educativa y al apoyo del entorno político-institucional local, dado que su papel no solo es relevante, sino que es determinante. El protagonismo debe extenderse a todas las personas y apoyar con mayor intensidad al núcleo de operaciones porque es en el contacto directo entre el profesorado, los profesionales, el alumnado y las familias donde se producen más tensiones o mayores satisfacciones. El cambio debe generar un sistema de aprendizaje continuado que se incorpore a la cultura organizacional bajo los principios que inspiran las organizacio- 
nes inclusivas que aprenden y emprenden (Álvarez-Arregui, Rodríguez-Martín, Agudo Prado y Arreguit, 2017).

\section{Lección XXIV}

Una cultura organizativa inclusiva debe y puede ser aprendida, porque se adquiere y transmite por la observación, la experiencia y el estudio; debe y puede ser compartida, porque afecta a todas las personas así como a su desarrollo personal, social y profesional; debe y puede cambiar las percepciones acerca de la educación porque incorpora mejoras y resuelve situaciones problemáticas; debe y puede tener capacidad de adaptación, ya que se basa en la capacidad evolutiva y adaptativa del ser humano; debe y puede ser transgeneracional porque se acumula y pasa de una a otra cohorte; debe y puede ser interinstitucional, porque pueden exportarse las buenas prácticas; debe y puede ser global porque la educación inclusiva puede extenderse a las localidades y a las ciudades a través de políticas integrales y desde las redes sociales; y debe y puede ser proyectiva si se enfoca hacia el futuro con intención de mejora (Álvarez-Arregui, 2002; Tonucci, 2009; Booth y Aiscow, 2015).

\section{Lección XXV}

Los errores que hemos encontrado de manera más generalizada cuando se plantean cambios en las organizaciones son: error psicológico (se enfatiza en los individuos sin tener en cuenta las relaciones de roles, normas y valores de la organización que conforman el sistema del cual forman parte); error sociológico (se piensa que los cambios en la estructura redundarán en un cambio de conducta de los miembros y que ese cambio se va a producir en el sentido planificado); error racionalista (se cree que hablando a las personas sobre la conveniencia del cambio se va a producir y se tiende a dar por sentado que un cambio justificado racionalmente redundará en el bien de una institución y se adoptará automáticamente.); error temporal (se produce cuando no se plantea el cambio en el momento adecuado, dado que hay otras causas que preocupan más a las personas, sean éstas problemas laborales, conflictos internos, exceso de burocratización, otros cambios concurrentes simultáneamente); y error cultural (se detecta cuando no se tiene en cuenta que en las organizaciones existen diferentes subculturas que deben gestionarse). Cuando no se actúa sobre las culturas de las organizaciones se acaban imponiendo unas sobre otras en un proceso de colonización cultural encubierto desde el que no se pueden fundamentar unos referentes sólidos para desarrollar un proyecto compartido que responda al tipo de institución que se quiere potenciar; al estilo de liderazgo que se quiere promover; al tipo de relaciones profesionales y con el entorno que se quieren desplegar; a las metodologías de aprendizaje-enseñanza que se quieren implementar; a las condiciones bajo las que se va a actuar; al grado de autonomía institucional que se quiere, se necesita y se va a utilizar; a las medidas que se van a adoptar para desplegar una educación inclusiva; y a la responsabilidad social que se asume con el entorno bajo los objetivos para el desarrollo sostenible que orientan la Agenda 2030. (Álvarez-Arregui, 2002, 2017; Álvarez-Arregui, Rodríguez-Martín, Agudo Prado y Arreguit, 2017).

Estas y otras lecciones, que se han aprendido y enseñado estos últimos años, no se han tenido en cuenta suficientemente en la construcción del Espacio Europeo de Educación Superior, de ahí que haya tenido y tenga que ser objeto de constantes revisiones que han puesto su atención en el diseño e implementación.
En los próximos años se deberá de poner mucho más énfasis en el aprendizaje y la enseñanza de valores comunes compartidos, lo que conlleva un importante proceso de reculturización en todos los ámbitos y niveles de responsabilidad de este ecosistema sociopolítico, jurídico, económico, laboral, tecnológico, de ocio, cultural y finito (Álvarez-Arregui, 2017; Arreguit, 2018).

\section{Un ecosistema de formación en nuestro entorno cultural: La Educación Superior en Europa.}

El momento actual, aunque a primera vista cabe calificarlo como complejo e incierto, puede ser propicio para iniciar procesos en las instituciones universitarias que encuentran dificultades para construir sus visiones y desarrollar estrategias que les permitan adaptarse al cambio continuo de manera más eficaz y afrontar los retos de futuro con mayores garantías de éxito. Los responsables de lo educativo son conscientes que en el formato actual estas organizaciones están abocadas a promover procesos de reestructuración y de reculturización internos sino quieren que sus misiones consolidadas, asociadas a la docencia y a la investigación, pierdan protagonismo y queden desplazadas como referentes educativos en las sociedades venideras.

Las universidades están viendo que el acceso generalizado a la tecnología, a los datos, la información, el conocimiento y el saber está imponiendo innovaciones continuadas y sistémicas que afectan a las competencias de las personas (saber, saber hacer y saber ser), sean físicas o jurídicas, por lo que el uso de plataformas intensivas, accesibles, versátiles y de bajo coste para la gestión, la investigación, la docencia y la transferencia están adquiriendo progresivamente mayor relevancia por el valor añadido que generan a los procesos y a los resultados. Esta evolución exponencial de la tecnología y del conocimiento las lleva inexorablemente a una sociedad dinámica donde una mayoría del alumnado ejercerá profesiones y utilizará herramientas que todavía no se han creado. Ante esta situación los currículums, los modelos de aprendizaje, los sistemas de selección del personal de administración y servicios, docente y de investigación, de evaluación de competencias, de estandarización, de certificación y de burocratización tal y como los conocemos, deben evolucionar (Álvarez-Arregui, 2018). La tarea no será sencilla porque son muchas las dimensiones y variables que se han ido entrelazando y emergiendo en un medio ambiente físico finito derivadas de la concurrencia del neoliberalismo (Bell, 1991; Gimeno Sacristán, 2001; Pérez Gómez, 1998), la globalización (Castells, 1999; Santos Guerra, 2000; Gairín, 1996), el desarrollo científico-tecnológico (Dosi, Friedman, Richard, Silverberg and Soete, 1990) y un acceso cada vez más abierto a los datos, la información, el conocimiento, la sabiduría, el aprendizaje, la enseñanza y la innovación (Drucker, 1969; Böhme y Stehr, 1986; Morin, 2001; Bolívar, 2000; Álvarez-Arregui, 2017; Xavier 2018).

El marco descrito, ver imagen, tiene recursiones directas e indirectas en los ecosistemas en los que nos integramos - sociales, laborales, de ocio, culturales, personales y políticos- actuando como referente, guía y apoyo porque son constructos que se retroalimentan constantemente.

El problema que nos encontramos es que las macrotendencias interactúan desde lo que se denominan "dinámicas no-lineales" y, debido a la evolución exponencial del desarrollo tecnológico, se provoca una aceleración superior en todos los ecosistemas concurrentes, lo que obliga a las personas físicas y jurídicas a una innovación continuada desde un aprendizaje y una enseñanza permanente. A este respecto conviene recordar a las universidades que, si bien han sido en los últimos siglos lugares de generación 
MEDIO AMBIENTE - FÍSICO - FINITO

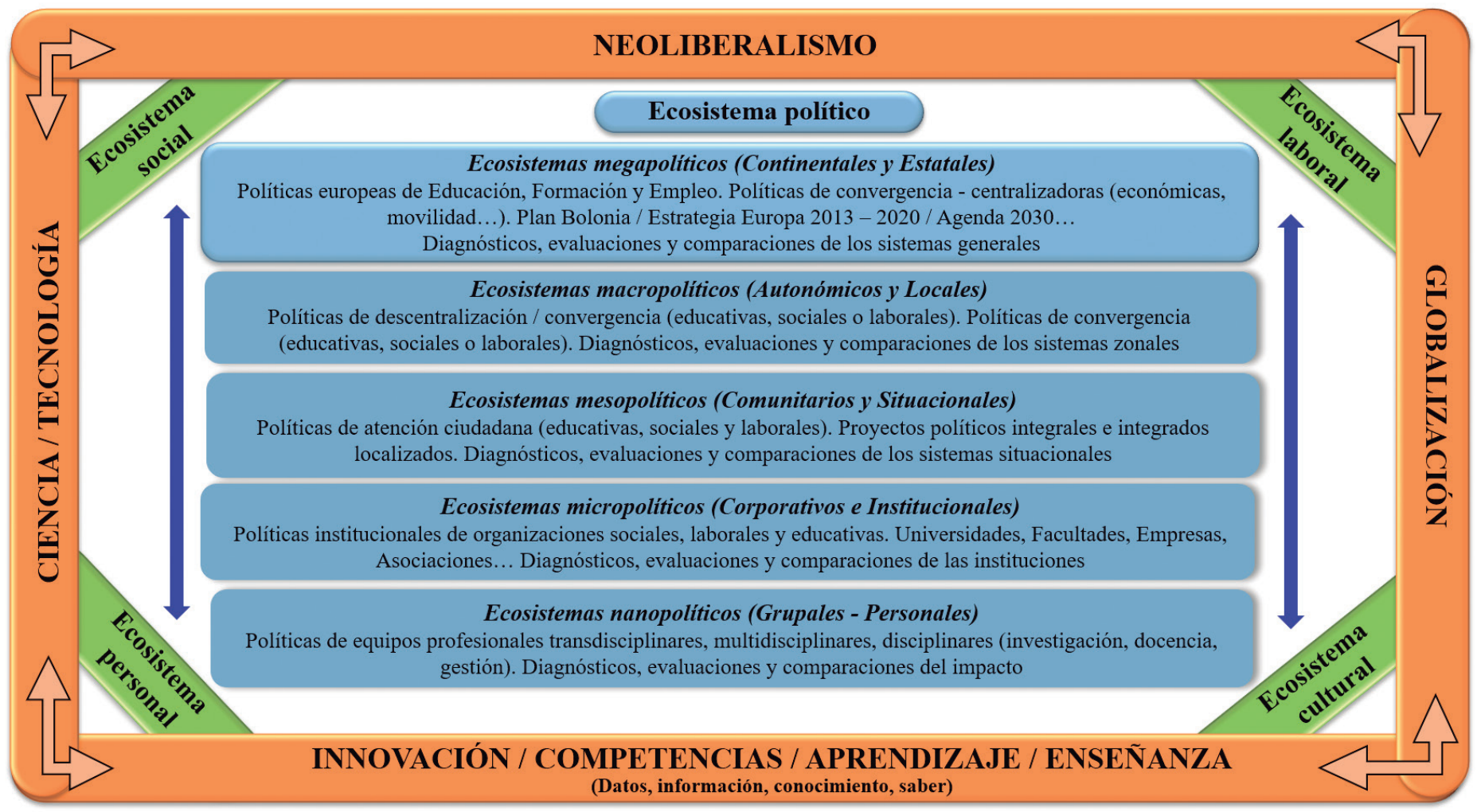

Figura 1. Visión ecosistema global y ejemplificación de los ecosistemas políticos en interacción dinámica

y de transmisión de conocimiento, deben reflexionar, entender y tomar decisiones sobre su situación actual en relación a la transferencia de conocimiento, sobre el uso que hacen de la tecnología, sobre su capacidad para anticipar futuras situaciones y sobre el valor añadido que generan sus procesos de transferencia de conocimiento para aportar soluciones viables para un desarrollo socioeconómico, político, jurídico y educativo sostenible en sus regiones de referencia, en particular, y en la sociedad, en general. Por tanto, el gran reto al que nos enfrentamos será capacitar a las personas físicas y jurídicas para sobrevivir en una realidad supranacional de fronteras difusas reequilibrando constantemente lo global con lo estatal, lo local con lo institucional y lo personal con lo público-privado. Este escenario hace necesario realizar diagnósticos precisos que permitan establecer acuerdos, vías de colaboración y compromisos explícitos entre todas las partes. De ahí que sigamos revisando algunas iniciativas de interés que se vienen planteando y otras en las que estamos trabajando dado que la construcción de ecosistemas universitarios que conjuguen un desarrollo profesional inclusivo en competencias genéricas y específicas ya no se pueden plantear de manera aislada, porque la enseñanza, el aprendizaje, el curriculum o la transferencia ya no es patrimonio de ninguna institución en concreto. Por tanto, la intervención debe adoptar un enfoque sistémico donde participen de manera responsable y comprometida todos los agentes con responsabilidad en lo educativo.

El modelo que se ha desplegado como válido en nuestro entorno cultural es el Espacio Europeo de Educación Superior (EEES), su desarrollo se viene implementando en las últimas décadas en los diferentes países a través de unos ejes que se adaptan situacionalmente y desde los que se pretende hacer frente a los retos actuales y de futuro de la Educación Superior. Es por ello por lo que se hace necesario tener en cuenta este marco como referencia de partida para cualquier modelo que se quiera promover porque integra los principios básicos a seguir por las instituciones universitarias y por lo tanto impregna, en mayor o menor medida, las visiones organizativas y funcionales que se despliegan en estas instituciones. A manera de recordatorio se presentan esquemáticamente:

\section{El primer eje plantea la necesidad de formar en y por competencias}

La literatura ha sido muy prolífica y controvertida en este punto y las corrientes de opinión se van desplazando pendularmente entre aquellos que van de posiciones de acatamiento acrítico hasta posiciones irreconciliables entre el enfoque por competencias y las pretensiones universitarias (Carabaña, 2011). En otras ocasiones han sido la investigación, el análisis y la conceptualización las que han guiado su desarrollo, orientándolo al mercado de trabajo (Alonso, Fernández y Nyssen, 2009). Sea como fuere el posicionamiento, el eje se ha ido incorporando a la normativa de los diferentes países comunitarios lo que ha generado una modificación de la planificación de los programas formativos que ha sido ardua y sigue inconclusa porque traspasa lo formativo (conceptos y procedimientos, saberes académicos) y adoptan una dimensión educativa transversal en la que se integran cuestiones axiológicas y actitudinales (saber ser) junto al saber y al saber hacer. Este planteamiento ya señalado por Delors (1996) ha sido tildado como inapropiado para la educación superior por algunos académicos que indican que devalúan el conocimiento; algo en lo que disentimos profundamente los que respaldamos el valor añadido que se deriva de lo educativo por el componente multidisciplinar que incorpora a la investigación, 
la docencia y la innovación. Además, consideramos que la libertad de cátedra no se ve alterada dado que deja margen para desarrollar los bienes intelectuales intangibles que la tradición universitaria siempre ha cultivado, si bien disentimos cuando se quiere restringir el potencial intrínseco de la competencia desde la medición, la cuantificación y la estandarización. En cualquier caso, respaldamos aquellos modelos que priorizan el aprendizaje a la enseñanza (Álvarez-Arregui, 2017; Gairín, 2003; González y Wagenaar, 2003; Rué, 2004) ya que otorgan mayor protagonismo a la persona y sitúan al docente como guía y facilitador de un proceso orientado a la consecución de unos objetivos claramente definidos. Además, es más permeable al trabajo en equipos multidisciplinares para el desarrollo de proyectos y revitaliza la tutoría, porque va más allá de la mera resolución de las dificultades derivadas de los programas, lo que requiere una formación inicial, continua, transversal y a demanda en función de las necesidades y expectativas que se planteen.

\section{El segundo eje hace referencia a la profesionalización y la em- pleabilidad}

La formación de profesionales siempre ha sido una de las funciones de la universidad, pero en la actual sociedad del aprendizaje y la enseñanza el cambio de perspectiva es evidente si se quiere poner el énfasis en el tipo de personas que se quiere formar. A este respecto venimos compartiendo aquellas iniciativas que presentan el EEES como "una buena oportunidad para introducir temas de desarrollo cívico y moral en los nuevos currículos para formar a los futuros profesionales de nuestra sociedad, que podrán actuar de forma responsable, libre y con compromiso" (Boni y Lozano, 2006: 11). A pesar de las buenas intenciones que subyacen en estas argumentaciones consideramos que están lejos de generalizarse, solo tenemos que revisar estos contenidos en los currículos universitarios para detectar que su importancia es testimonial. La consecuencia palpable es un distanciamiento progresivo con el auténtico ideal de educación donde no son tan relevantes los porcentajes de egresados especializados profesionalmente preparados, sino las actitudes y los valores (saber ser). Este hecho limita la voluntad política recogida en el Real Decreto 1393/2007 donde se planteaba que "la nueva organización de las enseñanzas incrementará la empleabilidad de los titulados (...)”. En este punto consideramos que sigue existiendo una desconexión entre la universidad y entorno laboral, por lo que deberá reforzarse con nuevos puentes de entendimiento, así como revisar el concepto de empleabilidad, ya que con el paso del tiempo se ha convertido en un desiderátum porque el mercado no es capaz de absorber al número de egresados que cada disciplina provee, lo que genera tensiones entre la formación universitaria y el empleo (Santos Rego, 2014). En cualquier caso, estos desequilibrios que se detectan entre formación y empleabilidad no pueden derivar en una ausencia de reconocimiento del valor intrínseco del conocimiento, sino que se hace necesario reconstruir el modelo poniendo más énfasis en la Formación Dual Universitaria, lo que vuelve a avalar una mayor implicación de los políticos, las entidades sociales, las empresas, los académicos y la ciudadanía (Álvarez-Arregui, 2017, 2018, 2019).

\section{El tercer eje se refiere a la investigación}

La producción del saber que caracteriza otra de las misiones de la Universidad se ha ido escorando hacia una alta especialización, es eminentemente aplicado y se está orientando hacia la transferencia y a la innovación con repercusiones más positivas para la gran empresa que para la mediana y pequeña. Este punto debe ser objeto de especial atención en los próximos años porque, tal y como nos indicaba Edgar Morín (2009: 44) la hiperespecialización impide ver lo global, el conjunto, de tal forma que con este planteamiento se pone la atención en lo esencial e imprescindible, se compartimentaliza el conocimiento y se disuelve. El problema lo encontramos en la práctica, ya que en ella las situaciones que requieren soluciones nunca son fragmentarias, por lo que se demandan enfoques pluridisciplinares para abordar los objetos de estudio en el contexto actual. The European Research Area tuvo en cuenta este déficit al "establecer una zona de investigación sin fronteras en que los recursos científicos se utilicen mejor al efecto de fomentar el empleo y la competitividad en Europa (...) combinando tres conceptos: la creación de un mercado interior de la investigación, reestructuración de la investigación europea, establecer una política europea de investigación" (Comisión Comunidades Europeas, 2000). Aunque ha habido avances en esta dirección desde el EEES, se están tomando decisiones que pueden tener efectos no deseados para el desarrollo de las personas físicas y jurídicas si se comete el error de burocratizar los resultados científicos o si se priorizan las iniciativas privadas avaladas por las grandes corporaciones en detrimento de los hallazgos de aquellas disciplinas que ofrecen claves de valor al ser humano. A pesar de que se vienen apuntando estrategias para el desarrollo de las Artes, las Humanidades y las Ciencias Sociales y Jurídicas nos seguimos encontrando una mayor resistencia de estas áreas de conocimiento al proceso de convergencia del EEES que desde las Ciencias Naturales y los campos técnicos (Pechar y Pellert, 2004). En cualquier caso, la internacionalización de la investigación y los resultados que de ella se deriva es en palabras de Scott (2006) una misión de la universidad que presta servicio a los estados-nación, por lo que no puede plantearse desvinculada de la docencia y del valor que aporta al servicio público nacional. Este planteamiento vuelve a avalar la necesidad de desarrollar proyectos de investigación pluridisciplinares que exigen forjar alianzas entre los políticos, las entidades sociales, las empresas, los académicos y la ciudadanía en el desarrollo de proyectos de investigación.

\section{El cuarto eje se asocia con la internacionalización y la movilidad}

La perspectiva sistémica y glocal vuelve a estar presente en este eje, ya que se da valor a las relaciones internacionales que se establecen entre instituciones, docentes, estudiantes y profesionales (Tennant, McMullen y Kaczynski, 2010: 6), lo que respalda un currículo abierto con una fuerte base internacional/universal. Este enfoque proporciona un abanico más genérico de competencias que favorece el perfil profesional de las personas con lo que incrementa su empleabilidad, pero además es susceptible de integrar la cultura del país nativo y proyectarlo al exterior y viceversa, con lo que está profundizando en los sentimientos de pertenencia comunitaria y de integración. En cualquier caso, hay que estar alerta sobre las posibles desviaciones, porque puede darse el caso de que se utilice como estrategia de competencia en el mercado global para atraer recursos humanos internacionales con lo que se podrían desvirtuar las pretensiones iniciales. También debe reflexionarse sobre la capacidad de las universidades para acoger colectivos internacionales y ofrecer programas formativos que estimulen la experiencia intercultural, profundizando en las dimensiones socioeducativas, académicas e investigadoras desde una perspectiva europea y mundial. Por último, será preciso reconocer que la homogeneización ha primado sobre la armonización, por lo que la pluralidad curricular se ha resentido como efecto de la estandarización de tal forma que el modelo 
único sigue corriendo el riesgo de no ser asumido para desarrollar las competencias que se van a necesitar en el espacio laboral europeo y también puede dar lugar a un incremento de las fricciones con las tradiciones universitarias en las que la formación liberal, la educación universal y la sabiduría siempre han tenido un lugar representativo (Barnett, 2005).

\section{El quinto eje se vincula a la dimensión socioeducativa y a la ciudadanía europea}

La Declaración de Bolonia (1999) demandaba una profundización en la herencia social y cultural de Europa, por lo que asumió un compromiso con la globalización, el desarrollo científico-tecnológico, las condiciones de mercado asociadas al neoliberalismo, el acceso compartido de datos, información y conocimiento lo que revitalizó el aprendizaje y la enseñanza a lo largo de toda la vida como eje transversal fundamental en el EEES. En este contexto, se han desplegado diferentes proyectos como la cátedra Jean Monet para evitar que los valores comunes compartidos superasen una concepción reduccionista de ciudadanía asociado al consumo, el rendimiento o el tratamiento mercantil del conocimiento desde la sociedad civil. En este punto es importante resaltar una vez más el valor de la glocalización, ya que esta metáfora permite hacer transferencias entre las identidades, expectativas, demandas y necesidades locales y globales favoreciendo la construcción de una ciudadanía europea donde las personas vean reflejadas sus raíces singulares, pero a la vez cosmopolitas donde la diversidad y los núcleos de significado comunes nos lleven hacia una auténtica educación cívica. En definitiva, compartimos con López-Gómez (2015) que el núcleo sustantivo de valores y propósitos que Europa ha asumido como tradición para el desarrollo del EEES debe ser un substrato común de hondo calado sino se quiere que sucumba ante un utilitarismo mercantil o ideológico.

\section{El sexto eje tiene en cuenta la competitividad y el aseguramiento de la calidad}

La competitividad y la calidad han sido dos pilares fundamentales en el proceso de convergencia europea. En la Comunicación de Salamanca (2001) ya se indicaba que la calidad no basta con que se dé, hay que poder demostrarla y garantizarla para que la reconozcan y aprecien los estudiantes, los responsables y toda la sociedad del país, de Europa y del mundo. Es por ello por lo que expresiones como rendición de cuentas- accountability-, partes interesadas- stakeholders-, aseguramiento de la calidadquality assurance- o performativity han venido ocupando un espacio representativo en las iniciativas del EEES.

Tennant, McMullen y Kaczynski (2010: 8) nos recordaban que la educación superior se ha visto cada vez más como un recurso que debe ser organizado para maximizar su contribución al desarrollo económico. Este hecho es importante porque las demandas a las personas físicas y jurídicas implicadas en la educación superior plantean exigencias a las universidades en base a las inversiones públicas que se hacen en ellas, de ahí que en las culturas políticas, académicas y sociales se hayan incorporado expresiones como accountability donde cobra un nuevo impulso la transparencia en el nuevo modelo de Gestión Pública.

En el caso de España, la ANECA y otras agencias de carácter autonómico llevan a cabo la actividad evaluadora, certificadora y acreditadora del profesorado; de las propuestas de planes de estudio en el EEES; del seguimiento y la acreditación de títulos oficiales; de la docencia que se desarrolla; de los sistemas de garantía de calidad interna en las titulaciones; y valora las menciones de excelencia en los estudios de doctorado, entre otras tareas. Estos procesos y acciones pretenden reflejar la eficacia de las prácticas al evaluar aquello que pretende ser objeto de medida con estándares identificados que hacen que la universidad se preocupe por lo que funciona y puede demostrarse (Pasias y Roussakis, 2012: 317). En este punto hay que estar de nuevo atento a las desviaciones que se generan ya que, como bien señala Power (1999), la institucionalización de comprobaciones a gran escala está dando paso a sistemas burocráticos perversos que están haciendo que se derrumben los cimientos de apoyo ante las contantes demandas de información, por la asignación de recursos a vigilancia o por el agotamiento y desmotivación del capital humano para desarrollar sus funciones docentes e investigadoras en unas condiciones de control latente y presente. Parece entonces que, pese a la seguridad que pueda proporcionar la evidencia y la evaluación de la calidad, se hace necesario recuperar el valor de la cultura, de la iniciativa y de los valores que han acompañado a la institución universitaria para construir un EEES.

En nuestra opinión la competitividad y la calidad se muestran insuficientes para construir una universidad del siglo XXI que aspire a integrarse y a ser protagonista en el proceso de cambio que acompaña el tránsito hacia una Sociedad del Aprendizaje, de la Enseñanza y del Conocimiento Global. Este propósito requiere un aprendizaje, enseñanza y formación continuada a partir del desarrollo de proyectos comunes donde participen de manera responsable representantes de las culturas académicas, políticas, sociales y empresariales. Es por ello por lo que, si se quiere apostar decididamente por un patrón de crecimiento socioeconómico sostenible, se debe fundamentar sólidamente $y$, para ello, se hace necesaria la creación de ecosistemas de innovación, emprendedores y creativos, con visión a corto, medio y largo plazo, con coordinación sectorial, desde la cooperación interinstitucional, con inversiones sostenidas con una mayor colaboración público-privada y con un compromiso explícito entre todos los agentes implicados.

La academia, la empresa, la administración pública, los políticos y los agentes sociales no pueden actuar de manera aislada y deben desarrollar estrategias de intervención compartida si su objetivo es desarrollar una innovación educativa sostenible fundamentada en una transferencia continuada del saber, del saber hacer y del saber ser entre los sistemas científico-tecnológico empresarial, académico, social y viceversa.

En nuestra opinión, cualquier ecosistema de formación/ aprendizaje que quiera orientarse a la adquisición y mejora de competencias para todas las personas debe orientarse al menos desde la racionalidad, la flexibilidad, la permeabilidad, la colegialidad, la profesionalidad, la multidisciplinariedad, la autogestionabilidad, la creatividad, la inclusividad, la transferibilidad y la complejidad. Este enfoque supone adoptar simultáneamente un enfoque global, local, institucional y personal (Álvarez-Arregui y Rodríguez-Martín, 2013, 2014, 2015) y se distancia de aquellas visiones disciplinares del currículum, porque va más allá de la consideración aislada de las personas, los objetivos, los contenidos, la evaluación o las experiencias y va integrando valor añadido gracias al Internet de las cosas, los procesos de automatización, el Big Data, la simulación de procesos o los valores que van impregnando el entorno global como los que emanan de la Agenda 2030.

Los ecosistemas de aprendizaje y enseñanza emprendedores se convierten así en una alternativa viable para dar respuestas a las demandas de una sociedad compleja ya que, desde ellos, se abordan aquellas cuestiones a las que se está dando una gran importancia en los entornos socioeducativos, económicos, culturales, de ocio, políticos, sindicales y laborales. A este respecto destacan las 
habilidades para gestionar el propio proceso de aprendizaje-enseñanza; disponer de elementos y metodologías para desarrollar procesos de aprendizaje-enseñanzae flexibles; comunicar y compartir conocimientos sin barreras lingüísticas y culturales; enriquecer los aprendizajes y las enseñanzas con la participación de profesionales de empresas, administraciones, ONGs, etc. y, finalmente, utilizar los espacios presenciales y virtuales en función de las necesidades para dotar de flexibilidad al modelo y favorecer la construcción de ecosistemas de aprendizaje y enseñanza que respondan a las demandas de competencias personales, profesionales, institucionales y comunitarias.

\section{Fundamentos básicos de una propuesta de cambio con ecosis- temas de aprendizaje y enseñanza}

En los últimos años venimos trabajando en el desarrollo de un modelo de formación ecosistémico (Álvarez-Arregui, 2010; Álvarez-Arregui y Rodríguez-Martín, 2015; Álvarez-Arregui, Rodríguez-Martín, Maldonado, Grossi y Arreguit, 2017; Álvarez-Arregui, Rodríguez-Martín, Agudo Prado y Arreguit, 2017) sostenible, abierto, flexible e inclusivo, atento a las necesidades, expectativas y demandas de las personas, las instituciones y el entorno. Es nuestro objetivo superar las limitaciones que plantean otros enfoques más rígidos, reduccionistas, burocráticos y mecanicistas en su conceptualización e implementación.

Nuestra propuesta tiene en cuenta otros modelos caso del el Ecosistema del conocimiento (Shrivastava, 1998); el Ecosistema e-learning basado en tecnologías y sistemas de gestión y apoyo al aprendizaje (Ismail, 2001); el Ecosistema modular (Dimitrov, 2001); el Ecosistema e-learning de trabajo y aprendizaje (Wilkinson, 2002); el Ecosistema e-learning para profesionales (Brodo, 2006), y el Learning-Ecosystem de Gült y Chang (2009), entre otros. En estos modelos se incorporan entre otros componentes un diseño de aprendizaje, una población (profesorado, estudiantes, agentes educativos, etc.) y un sistema de formación para el desarrollo de competencias básicas y aplicaciones de las webs 2.0, 3.0 y 4.0. El peligro latente que detectamos en estas propuestas es que se pueden acabar deslizando, quizás en exceso, hacia el e-learning únicamente (Uden, Wangsa y Damiani, 2007), por lo que nos parece más aconsejable situarse en un aprendizaje mixto situacional (blended situational learning) para aprovechar las potencialidades que nos brinda el desarrollo tecnológico continuado para generar espacios de aprendizaje presenciales y virtuales que integren a los diferentes colectivos concurrentes, cubriendo todas las posibilidades posibles en el espacio presencial y virtual (de arriba-abajo, de abajo-arriba, lateralmente y transversalmente), incorporando sistemas (de tutorización, de asesoramiento, de autogestión del aprendizaje, de gestión de datos, información, conocimiento y saberes...), utilizando bases de recursos propios y ajenas donde aprendemos a hacer, aprendemos a innovar, aprendemos a aprender, aprendemos a transferir y aprendemos a ser compartiendo valores (Álvarez-Arregui, 2017; Álvarez-Arregui, Rodríguez-Martín, Agudo Prado y Arreguit, 2017).

En este contexto somos conscientes de que un modelo se puede diseñar desde diferentes enfoques en función de la perspectiva que se adopte, ya que son muchos, los parámetros, las coordenadas, los factores y las variables que concurren situacionalmente tal y como se ha comentado en las referencias anteriores. Es por ello por lo que se presentan de manera sintética aquellos principios fundamentales que vienen guiando nuestro trabajo en los últimos años (Álvarez-Arregui y Rodríguez-Martín, 2014, 2015, 2016; Álvarez-Arregui, Rodríguez-Martín; Madrigal Maldonado, Grossi -Sampedro y Arreguit, 2017):
Una visión y unos valores compartidos. En un EAECE (Ecosistema de Aprendizaje y Enseñanza Creativo y Emprendedor) se tiene que consensuar y compartir una visión de tal modo que integre las diferentes subculturas de las que participan los distintos profesionales y que sea coherente con unos objetivos comunes que sean beneficiosos para todas las personas.

Un liderazgo distribuido. En un EAECE los diferentes profesionales y personas concurrentes tienen que tener oportunidades de desarrollo personal y profesional de tal modo que se potencie su capacidad de liderazgo, de autogestión, de trabajo en equipos multidisciplinares, de desarrollo de proyectos creativos, de comunicación de iniciativas orientadas a la mejora del aprendizaje y de un mejor desempeño de las funciones asignadas a su puesto de trabajo. El liderazgo deberá ser situacional combinando la vertiente educativa, transformacional, delegada, inclusiva, sostenible y tendrá proyección en las personas, la institución y la comunidad.

Un aprendizaje individual, colectivo, organizativo y abierto. En un EAECE se deben identificar las potencialidades de las personas, de los equipos y de la organización para generar sinergias dentro y fuera de la institución. Es importante detectar qué necesitan, qué quieren aprender, en qué tienen que formarse y cómo deben de hacerlo para generar conocimiento, gestionarlo y compartirlo. Este planteamiento impulsa al ecosistema, porque lo hace crecer a través de la construcción de un tejido socioprofesional multidisciplinar de aprendizaje y enseñanza, donde la confianza y la mejora continua revitaliza la capacidad de la organización para diagnosticarse, planificar, implementar y aprender a emprender desde la formación continua, la creatividad, la solución de problemas, el desarrollo de proyectos, la consolidación de equipos de trabajo y el fortalecimiento de las redes soicioemocionales intra e interinstitucionales.

Una orientación de los procesos hacia el desarrollo sostenible. En un EAECE la práctica profesional deja de ser algo privado para pasar a ser de dominio público. Las observaciones, los registros, los grupos de discusión, los foros, los documentos institucionales, los protocolos y las experiencias previas serán algunos elementos que se utilizarán para compartir, para reflexionar y para mejorar la práctica, lo que garantiza que aprendizaje y la enseñanza sean pragmáticos, colaborativos, motivadores, abiertos y transformadores.

Una promoción de la confianza, el respeto, la negociación y el apoyo mutuo como base de los sistemas de relaciones. En un EAECE todas las personas deben sentirse apoyadas, valoradas e integradas, ya que cuando así sucede se genera confianza y se asumen compromisos con los proyectos y los procesos de mejora que se promuevan. Las diferencias individuales, la disensión y el error son aceptados dentro de una reflexión crítica que promueva el desarrollo del equipo, no existiendo en principio dicotomía entre individuo y colectividad.

Una proyección exterior a través de redes y alianzas interinstitucionales para compartir e intercambiar información y conocimientos. En un EAECE se favorece la intradisciplinariedad, la interdisciplinariedad y la transdisciplinariedad, promoviendo sistemas de relaciones que transcienden los límites de los equipos, de los proyectos y de la organización. Las disensiones se negocian, se construyen lazos y vínculos sólidos entre los equipos, entre los equipos y la institución, y de todos ellos con los entornos en los que se proyecten y con los que colaboren. En este escenario, los ecosistemas crecen y se desarrollan a través de redes profesionales, personales e institucionales, lo que deriva en espacios abiertos donde fluye la información y 
la comunicación y se genera aprendizaje y conocimiento que repercute en el crecimiento de las personas, de los equipos y de la institución a lo largo de todo el proceso de innovación.

Un compromiso y una responsabilidad social con el entorno. En un EAECE se asumen compromisos con la mejora de los equipos, de la institución y del entorno. Se valora que se afronten riesgos cuando se promueven iniciativas y se diluye el temor a sufrir críticas ante los errores, ya que se convierten en fuente de aprendizaje. Se invita a buscar apoyos cuando emergen problemáticas sociolaborales y se avala que se compartan los éxitos personales, profesionales, institucionales y comunitarios, así como la transferencia de mejoras al entorno local, en particular y a la sociedad, en general.

La colaboración como pilar organizativo, funcional y de aprendizaje de valores. En un EAECE se garantizan ciertos elementos de orden físico, estructural, económico y emocional de modo que se aseguren las condiciones espaciotemporales de colaboración y un aprendizaje compartido. En esta dirección, deben proveerse recursos tales como materiales de trabajo, información, asesoramiento, material multimedia, protocolos $\mathrm{u}$ otros elementos que se determinen como necesarios para favorecer el aprendizaje y la enseñanza en el ecosistema.

En último término, un EAECE se perfecciona constantemente en sus diferentes fases, ver figura, a través del diagnóstico, la cre-acción, la planificación, la implementación, la evaluación, la transferencia, y la investigación sobre el impacto. En ese proceso, el EAECE es capaz de descubrir nuevas ideas, de integrarlas y de transfórmalas orientándolas hacia sus objetivos. Por tanto, tiene capacidad de aprender y enseñar a aprender, de aprender y enseñar a mejorar, de aprender y enseñar a adaptarse y de aprender y enseñar a crecer de manera sostenible.

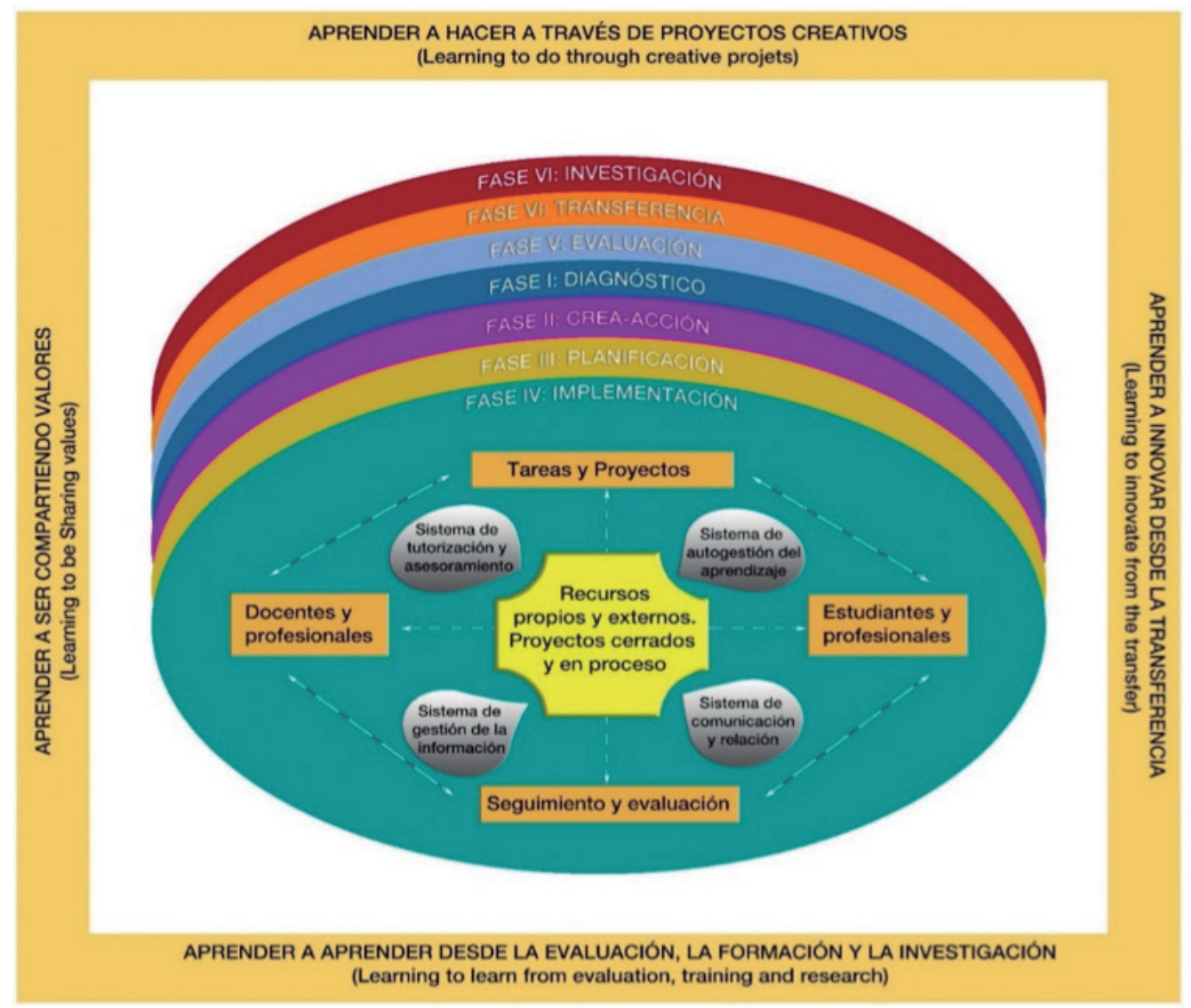

Figura 2. Ecosistema de aprendizaje-enseñanza creativo y emprendedor EAECE: Marco, fases y referentes.

Atendiendo a los referentes citados, continuamos trabajando en el desarrollo de un ecosistema de aprendizaje - enseñanza universitario que, a manera de plataforma de aceleración de la innovación educativa, se está integrando de manera transversal en los ámbitos educativos, sociales y laborales de la Universidad de Oviedo a través de proyectos de innovación, de investigación y de transferencia educativa. La forma administrativa que ha adoptado es la de Instituto de I+D+i Educativa, (INIE) que evoluciona constantemente para adaptarse a las diferentes situaciones que van emergiendo en la Universidad de Oviedo.

Este ecoentorno de aprendizaje y enseñanza integra en su diseño, estructura y funcionamiento los principios y procesos pre- sentados lo que queremos transmitir a las personas y organizaciones con las que interactuamos. Otra característica importante es su adaptabilidad, ya que es susceptible de ser replicado en diferentes contextos en función de las demandas, expectativas y necesidades de organizaciones, instituciones o equipos con las que interactuamos. Siendo coherentes con nuestra visión, damos gran importancia al análisis inicial ya que debe captar la complejidad de los componentes del sistema y asumir la necesidad de integrar las dimensiones social, educativa, laboral y política de los componentes concurrentes en cualquier proyecto de intervención. Por tanto, el punto de partida se fundamenta en un diagnóstico de los recursos disponibles en cuanto a los ele- 
mentos físicos (instalaciones, herramientas, conocimiento, saber hacer...), los elementos humanos (perfiles de profesores, de estudiantes, de profesionales, de asesores, de usuarios..., la financiación, los recursos disponibles, las visiones, las estrategias, las expectativas de los promotores y las actitudes de confianza y colaboración necesarias para integrar las culturas académicas, políticas, sociales y empresariales. A partir de aquí se va crean- do una visión desde un enfoque dialógico en el que se Aprende y se Enseña a Aprender y a Enseñar, a Colaborar, a Emprender y a Aceptar el Cambio.

La misión, la planificación estratégica, la colaboración y la evaluación continua son garantes de la transparencia de los procesos y permiten articular procedimientos para revisar y reestructurar regularmente la visión.

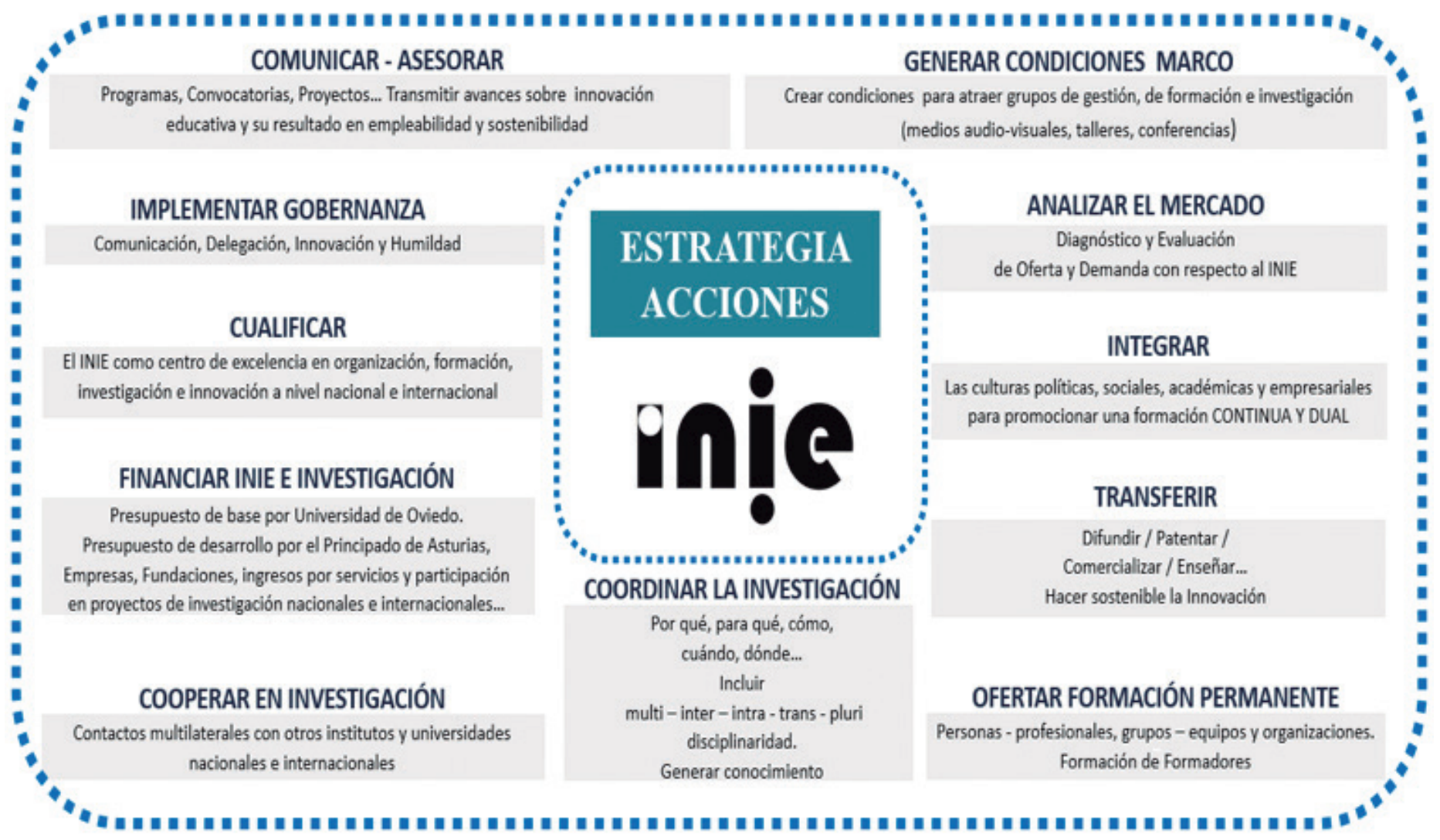

Figura 3. Estrategia y acciones del INIE para promover la I+D+i Educativa

Las acciones que se promueven a partir de estos referentes van generando valor añadido a las personas (usuarios, profesionales y comunidad), a las metodologías, a las herramientas, a los protocolos, a las tecnologías, a los sistemas y a las instituciones.

El objetivo final es generar Innovación Educativa si bien somos conscientes que ésta se puede producir en cada una de las acciones, de manera combinada o de manera global. Por tanto, no se plantea una relación lineal causa-efecto entre las acciones, sino que el proceso de cambio se entiende como circular, sistémico y atemporal donde se plantea el aprendizaje y la enseñanza como un continuo en el que tienen que participar las partes interesadas, a saber, académicas, políticas, sociales, laborales y empresariales. A manera de ejemplo, presentamos las acciones que estamos promoviendo actualmente desde el INIE, ya que ejemplifican con bastante claridad a lo que nos referimos, ver figura 4 (Álvarez-Arregui, Rodríguez-Martín, Agudo Prado y Arreguit, 2017).

Estas acciones se integran en proyectos que se pueden coordinar por una o varias entidades y adquieren todo su potencial cuando las hélices integran el conocimiento y la experiencia de las culturas del entorno sociopolítico, académico, empresarial y de la Administración Pública. Los proyectos que se despliegan pretenden dar respuestas, creativas y coherentes a la ciudadanía a través de un trabajo multidisciplinar que afecta positivamente al desarrollo personal, profesional, institucional, local, regional, nacional e internacional en función de la proyección que se le quiera dar Álvarez-Arregui, Rodríguez-Martín, Madrigal Maldonado, Grossi-Sampedro y Arreguit, 2017).

Para cerrar este punto presentamos una visión integrada del ecosistema que estamos desarrollando, ya que permite clarificar las argumentaciones anteriores donde se recogen de manera sintética múltiples aportaciones de la teoría, la práctica y la experiencia. Como puede verse en la figura 5, la estructura general se fundamenta en cuatro componentes básicos de referencia, un entorno, una población, un sistema de relaciones y una tecnología. Asociados a cada uno de ellos aparecen unos elementos de referencia que son susceptibles de múltiples combinaciones y desarrollos en función de los proyectos, objetivos y prioridades que se hayan establecido. En la parte central aparecen la orientación del aprendizaje y la enseñanza que se genera a través de los proyectos que se promueven, los valores que los guían y las personas, áreas y espacios geográficos sobre los que se proyecta. 


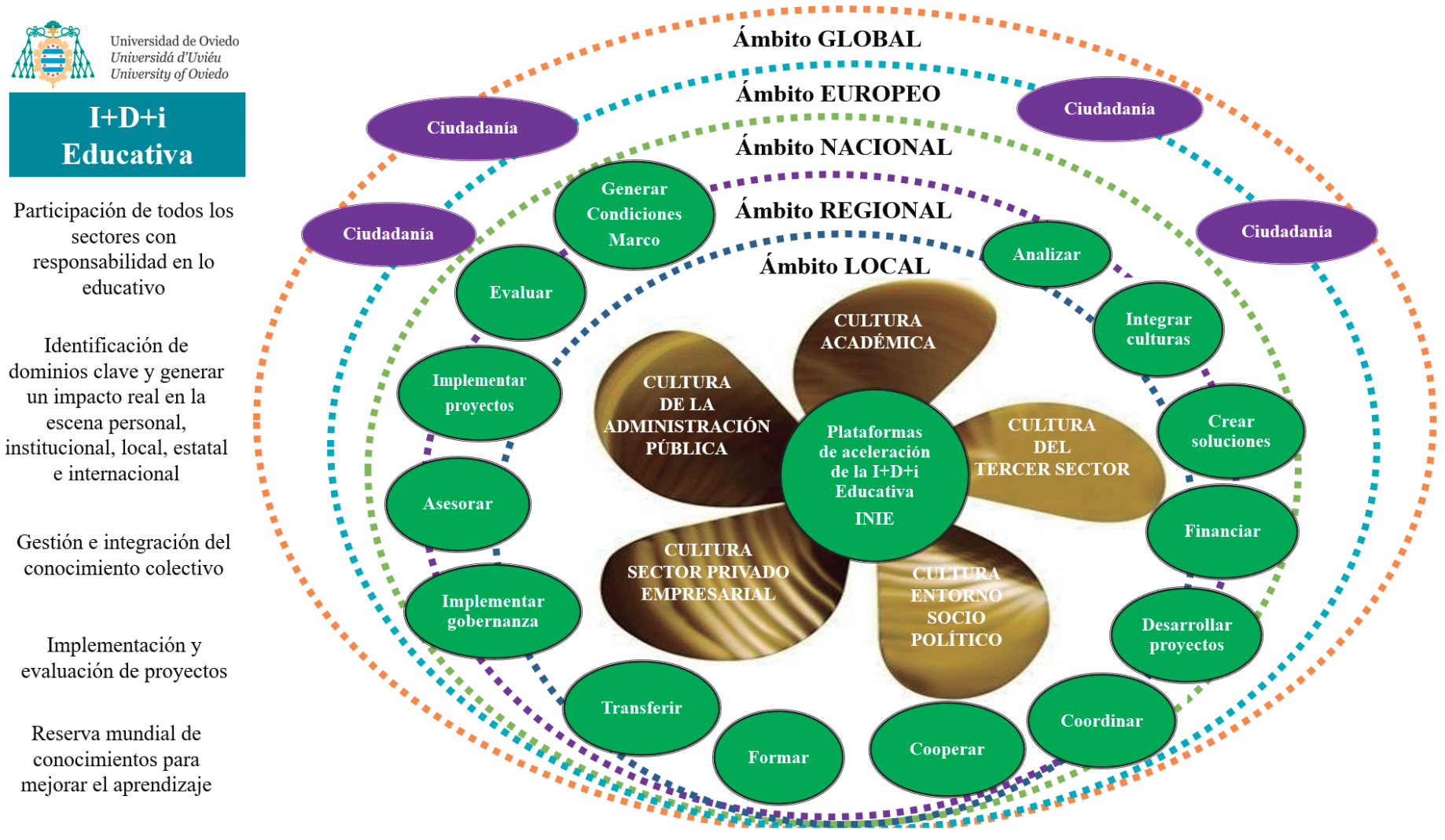

Figura 4. Proyección glocal del INIE entendido como plataforma de aceleración de la Innovación Educativa

Por el componente población entendemos las personas físicas y jurídicas que concurren en un entorno cultural y espacial determinado, formando un ecosistema donde los actores como el alumnado, al profesorado, las familias, los asesores externos, el personal de administración y servicios, la administración local, los responsables y profesionales de distintas entidades políticas, sociales, educativas y laborales son elementos para considerar en función del proyecto. La población tiene sus propios axiomas (Hawley, 1982) así puede hablarse de niveles óptimos o desajustados en función del número de participantes, de un tamaño más o menos adecuado en función del grado de especialización que se requiera o de espacios y tiempos más o menos apropiados en base a la tecnología o los sistemas que se quieran establecer, entre otras cuestiones.

Los sistemas de interacción que se establezcan en un ecosistema son fundamentales para cumplir sus objetivos y serán los que determinen la estructura singular del mismo. Estas relaciones se presentan de forma estática cuando nos refiramos a los organigramas organizativos o a los sistemas de relaciones formales y se plantearán como dinámicas cuento se consideran los sistemas de relaciones informales, ad hoc, de formación de asesoramiento de investigación o de innovación. Como es lógico las relaciones no van a ser simbióticas (colaboración) en todos los casos, sino que habrá sistemas de poder que se irán estableciendo en base a los intereses de los participantes por lo que emergerán conflictos que se abordarán como un proceso más de aprendizaje y enseñanza. También abordamos del mismo modo los diferentes niveles de competencia, las divergencias ideológicas, las emociones, la experiencia o la dependencia.
Los ecosistemas necesitan energía para sobrevivir por lo que necesitan disponer de herramientas pertinentes y singulares para captarla y transformarla. En este contexto nos parecen acertadas las argumentaciones de Duncam (1959: 689) cuando se refiere a la tecnología en la ecología humana "... no sólo a un complejo de arte y artefactos cuyas pautas son inventadas, difundidas y acumuladas..., sino a un conjunto de técnicas empleadas por una población para ganar el sustento de su medio ambiente y para facilitar la organización de la actividad productora del sustento. En la imagen pueden verse algunos proyectos habituales asociados a la tecnología en entornos socioeducativos si bien éstos cambiaran en base al tipo de proyecto, la población y el entorno. En este último, distinguimos entre las estructuras tangibles (infraestructuras, espacios, tiempos, recursos y materiales), las intangibles caso de los espacios virtuales (personales interpersonales, administrativos, académicos, políticos, empresariales...), las simbólicas (asociadas al clima y las culturas que se generan en las organizaciones) y las políticas que vienen determinados por los sistemas de liderazgo que se establecen en distintos niveles.

Este enfoque muestra la complejidad del ambiente, lo que conlleva interpretarlo más allá de un mero continente para el desarrollo de acciones educativas, curriculares, formativas o investigadoras ya que se convierte él mismo en objeto de estudio, aprendizaje, enseñanza y formación. Es por tanto, fundamental tener en alta consideración este componente para el desarrollo de proyectos y acciones educativas, ya que es determinante para promover el cambio cultural que puede conceptualizarse, analizarse, reconstruirse y mejorarse. 


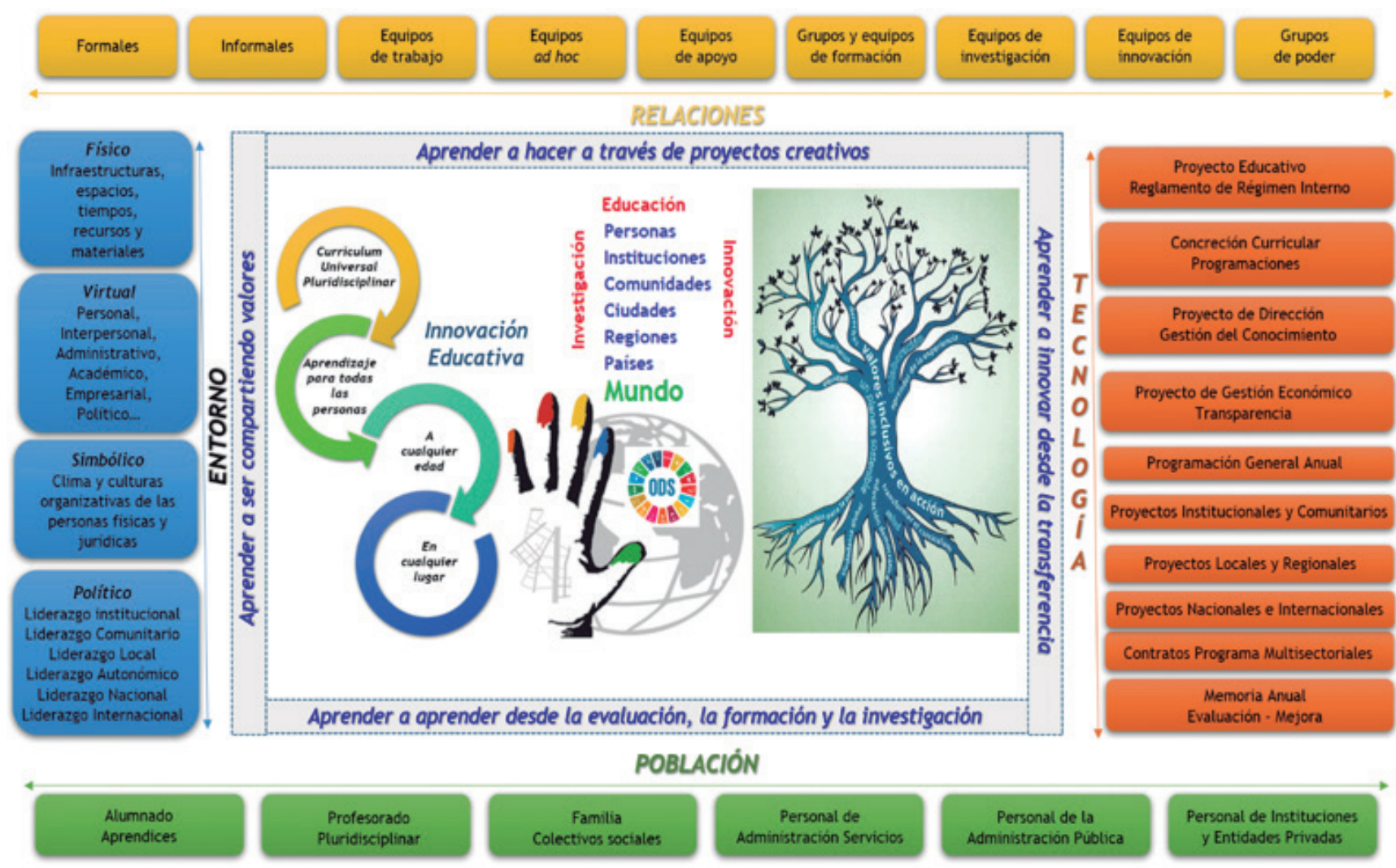

Figura 5. Visión general del INIE entendido como Ecosistema de Aprendizaje y Enseñanza Creativo y Emprendedor (Adaptado de Álvarez-Arregui, 2017).

En la parte central de la imagen se recogen los principios y valores que guían el desarrollo de los proyectos en este modelo de innovación educativa, así se integran, entre otros, los principios de las ciudades educadoras, el Índice para la Inclusión y los Objetivos de la Agenda 2030 para el Desarrollo Sostenible donde las universidades juegan un papel esencial. El currículum se diseña teniendo en cuenta los principios que guían el Diseño Universal de Aprendizaje (DUA), será pluridisciplinar y favorecerá el aprendizaje de todas las personas, a cualquier edad y en cualquier lugar.

Las competencias genéricas y específicas se abordarán, se aprenderán y se enseñaran a través de metodologías activas blended-learning con el objeto de promover un cambio actitudinal y cultural donde la búsqueda de alternativas, la solución de problemas, la utilización de nuevos servicios, la implementación de productos o el manejo de una metodología se fundamentará en un proceso sistemático y sistémico de capacitación continua donde los participantes aprenderán y enseñarán a hacer a través de proyectos creativos, aprenderán y enseñarán a innovar desde la transferencia, aprenderán y enseñarán a aprender desde la evaluación, la formación y la investigación y aprenderán y enseñarán a ser compartiendo valores y manifestándolos en la práctica profesional y social.

Proyección hacia el futuro: Ecosistemas de formación continua para una sociedad de aprendizaje y enseñanza sostenible y responsable

Ante una realidad compleja por multidimensional y dinámica, las soluciones simples, excluyentes o generalizables ya no son viables. La Unión Europea es consciente de ello y desde la Agenda 2030 para el Desarrollo Sostenible ha avalado un com- promiso internacional y un calendario para hacer frente a los retos sociales, económicos y medioambientales de la globalización, poniendo como objeto de atención a las personas, el planeta, la prosperidad y la paz, bajo el lema de "no dejar a nadie atrás". Desde la Universidad de Oviedo, en general, y desde el Instituto de Investigación e Innovación Educativa (INIE), en particular, consideramos que es una gran oportunidad para la mejora movilizar a todas las administraciones, a nivel nacional, autonómico y local, a la ciudadanía, a los actores sociales, a las empresas, a las universidades, a los centros de investigación y a las organizaciones de la sociedad civil, alrededor de una visión compartida que se extienda dentro y fuera de nuestra institución.

Somos conscientes que el desafío es enorme, pero inaplazable y urgente, lo que requiere liderar políticas en los diferentes ámbitos de responsabilidad ante las múltiples manifestaciones de pobreza, desigualdades, desempleo y degradación de nuestro medio natural que están emergiendo, por lo que todos los actores deben de estar implicados sin excepción. Por tanto, el desarrollo e implementación del modelo ecosistémico de aprendizaje y enseñanza creativo y emprendedor que hemos presentado es un claro compromiso con los principios y acciones que guían la Agenda 2030. Este referente supone una oportunidad para reforzar la misión educativa de la Universidad en la plena realización de los derechos humanos, la paz, el ejercicio responsable de la ciudadanía local y global, la igualdad de género, el desarrollo sostenible y la salud.

Los contenidos de dicha educación deben contemplar aspectos cognitivos, procedimentales, actitudinales y valores tal y como planteamos en los principios que guían los ecosistemas de aprendizaje y enseñanza creativos y emprendedores. Es importante, por tanto, que el modelo responda a los compromisos adquiridos en el marco de los ODS, preparando a las futuras cohor- 
tes de aprendices y enseñantes para vivir en un mundo complejo e interconectado. Por tanto el currículo debe ser abierto para incorporar contenidos, acciones y valores que permitan dotar a las personas de las herramientas y competencias necesarias para comprender el mundo y para manejarse en él con una capacidad crítica orientada por valores compartidos. En este marco también consideramos, al igual que la Organización Internacional del Trabajo (OIT), los cuatro pilares sobre los que debe fundamentarse un Programa de Trabajo Decente - creación de empleo, protección social, derechos en el trabajo y diálogo social. Estos referentes son elementos fundamentales para alcanzar los ODS y esenciales para que sean sostenibles e inclusivos los patrones de crecimiento socioeducativo y económico. Estas y otras cuestiones se han tenido en cuenta en el diseño, desarrollo e implementación de nuestro ecosistema y, más concretamente, se han integrado en el Instituto de Investigación e Innovación Educativa de la Universidad de Oviedo al igual que en otros ecosistemas internacionales con los que colaboramos a través de múltiples convenios y acuerdos. En nuestro caso estamos convencidos que desde este modelo se irán generando nuevas miradas, visiones, misiones y estrategias de las que participará cada vez más la ciudadanía y los actores de los entornos socio-jurídico-político, académico y económico, e iremos superando las limitaciones y problemáticas situacionales actuales y nos proyectaremos al futuro con mayores garantías de éxito.

En nuestra opinión, ya no es posible seguir avalando desde la Universidad el mantenimiento de sus estructuras organizativas actuales, donde la docencia, la investigación y la transferencia se rijan por indicadores culturales no alineados con las grandes nacrotendencias emergentes y los principios que guían la Agenda 2030. Estas instituciones también deben revisar sus sistemas de contratación de personal, porque ya no es asumible que se sigan incorporando profesionales con baja capacitación para la gestión, la docencia o la investigación, o adopten una actitud negativa ante la formación continua o que sigan impartiendo cursos de "base" de manera continuada sin modificar los contenidos a lo largo de los años. También reconocemos que no es viable seguir trabajando en unos entornos laborales donde la intensificación del tiempo de trabajo se ha convertido en una constante como consecuencia del desarrollo tecnológico y el incremento de la burocratización, pero eso no es excusa para seguir transmitiendo contenidos encapsulados en disciplinas, validados en base a criterios corporativos o individuales, sin control externo. La certificación burocrática de competencias a través de evaluaciones periódicas estandarizadas que no guardan correspondencia entre lo que se dice que se hace, lo que se dice que hay que hacer, lo que se hace realmente y los resultados que se obtienen, entre otras cuestiones, es otro problema importante que habrá que resolver.
Si los profesionales de la educación, los políticos, los aprendices y los empresarios se siguen orientando bajo estos referentes ni serán capaces ni podrán comprometerse con la formación, la investigación y la transferencia que requiere una sociedad del aprendizaje y la enseñanza continua. En cambio sí se adopta una actitud positiva ante el cambio en la Universidad, se aumenta el número de profesionales externos con contratos laborales temporales procedentes de la industria, de las administraciones públicas o de los sectores de servicios y tecnológicos y se buscan equilibrios y colaboración entre todas las partes podremos ir construyendo el mejor currículum posible, combinando los saberes institucionales avalados por la libertad de cátedra junto a los últimos saberes que se generen en los entornos laborales, académicos, sociales, jurídicos y políticos.

En este nuevo contexto de aprendizaje y enseñanza creativo y emprendedor tendrá cabida la necesidad creciente del profesorado para trabajar de manera transversal en sus clases para permitir que el alumnado no solo aprendan y enseñen materias, sino que tendrán que saber relacionarlas para poder comprender la complejidad de las temáticas que se abordan, lo que los llevará necesariamente a la pluridisciplinariedad y a una mayor eficiencia en los trabajos transdisciplinares. Por tanto, compartimos aquellas iniciativas que profundizan en el pluralismo estructural, funcional y axiológico, superando las limitaciones derivadas de los modelos burocráticos, competitivos y estandarizados, para ir integrando lo mejor de lo público, lo privado y el voluntariado a través de una mirada ecoformadora, pluridisciplinar, emprendedora, inclusiva e innovadora.

La no intervención ya no es una opción porque, de no asumirse compromisos, las controversias, las contradicciones, las paradojas, las polémicas y los enfrentamientos se seguirán incrementando al igual que las brechas de alfabetización y de capacidad de acceso a los recursos para las personas físicas y jurídicas en los diferentes lugares del planeta. Somos conscientes de que el proceso de cambio es de hondo calado porque es cultural.

Queremos concluir indicando que la excelencia universitaria se construye y se construirá con la participación activa y el compromiso de todas las personas y organizaciones, ya que desde esas plataformas se podrán ir creando vínculos interactivos entre el entorno natural, social, empresarial, político, tecnológico y académico, lo que hace necesario generar ecosistemas de aprendizaje creativos, innovadores inclusivos, interconectados, infoaccesibles e innovadores que mejorarán la calidad de vida de todas las personas a través de la I+D+i Educativa. Este enfoque nos permita avanzar hacia una coexistencia humana que garantice un futuro durable, deseable y sostenible a las generaciones actuales y futuras. 


\title{
The future of the University and the University of the Future. Ecosystems of continuous training for a learning and teaching society sustainable and responsible
}

\author{
Emilio Álvarez-Arregui $\left(^{*}\right)$ y Xavier Arreguit $\left({ }^{* *}\right)$ \\ ${ }^{*}$ ) Universidad de Oviedo (España) \\ (*) Innobridge S.L. (Lausanne-Suiza) \\ Emilio Álvarez-Arregui
}

Professor of the University of Oviedo in the Department of Education Sciencies, currently part of the institutional team as director of the Area of the Institute of Research an Educational Innovation. He has been principal researcher in regional, national and international projects and is currently working on the development of competency models with universities, research institutes, companies, administrations, counseling, social agents and platforms for accelerating innovation from different American and European countries. It has more than two hundred publications in magazines and books relative to these and other topics. He has made teaching and research stays in Spanish universities (Seville, Huelva, León, Balears Islands, Jaén, UNED...) and foreigners (Uruguay, Chile, México, Latvia, Portugal, USA, United Kingdom, Ireland, Brazil, Switzerland...). He is member of "the Spanish Society of Pedagogy", the "Universities and Inclusive Education" Group, the "Spanish Service for the Internationalization of Education", of the "European Network of Inclusive Education and Disability Included"; "European Forum of Education Administrators", "Transdisciplinary Educational Research Network"; "Inclusive University Network, Network of Educational Research Institutes of Spain", "Alfamed Euro-American Network"; "Ibero-American Network for the Evaluation of the Educational Quality of Higher Education"; of "the University Network for Postgraduate Studies and Permanent Education"; and "the UNESCO Network in the Principality of Asturias", among others. He is director of the "Aula Abierta" magazine.

email: alvarezemilio@uniovi.es

\section{Xavier Arreguit}

Managing Director and President of Innobridge SA and Innobridge Services Sàrl. He holds a MS Degree in Engineering and a PhD from the Swiss Federal Institute of Technology EPFL, and he subsequently obtained an Executive MBA at HEC-Lausanne / EPFL. After a postdoc in the Computer and Neural Sciences Department at the California Institute of Technology (CALTECH) in United States, he worked at the CSEM in Neuchâtel (where he was vice-president, in charge of Strategic Marketing and Business Development). In 2004, he co-founded Innobridge SA, now a holding company, and contributed in 2005 to the creation and management of JADE Invest SA, a subsidiary of CSEM, specialized in investing in start-ups. In 2010, he co-founded Innobridge Services Sàrl, a strategy and management consulting company with innovation expertise in Smart Cities, Industry 4.0, Cleantech, Sport, Medtech and Education. He has also contributed to several patents and products and to the creation, financing, development and sale of several start-ups. He has developed expertise in systems and innovation models involving public-private partnerships and has participated as an invited external lecturer in several master courses. He is a member of several Companies' Boards of Directors in Switzerland and in Malaysia.

email: xavier.arreguit@innobridge.com

\begin{abstract}
Today's society is characterized by its complex, interdependent, dynamic and informational nature, in which individuals and legal entities that are integrated into a political, social, economic, ecological, physical-finite environment and with limited resources concur. In this context, different macro-trends are temporarily and interdependently consolidated, accelerating processes exponentially in all areas. This leads to the emergence of new economic, political and social models that require people to permanently adapt their skills, which are necessary to avoid generating polarization, exclusion and insurmountable literacy gaps.

Universities that have been places of generation and transmission of knowledge must understand, accept and adapt to the emerging context and anticipate future situations, since if they do not do so, their missions will become irrelevant and they will be displaced as educational agents and references. In this article, we take a look at all these questions and propose an ecosystem model for training and learning contextualised to the new demands of the European Higher Education Area, taking into account the principles that guide the Agenda 2030 for Sustainable Development and which is being materialised in the Institute for Educational Research and Innovation (Spanish acronym: INIE) of the University of Oviedo through educational research and innovation projects and transversal training programmes.
\end{abstract}

Keywords: learning, teaching, training, ecosystem, educational change and innovation. 


\section{Contextualization}

The close connection that has been established between Environment, Society, Economy, Legislation, Knowledge, Technology, Work, Leisure and Education in the living environment of individuals and organizations, makes it necessary that public and private managers are having to develop new visions, missions and glocal strategies to establish adjustments to the imbalances that emerge in the system and the subsystems as a result of the concurrence of macro-framework trends and concurrent situational variables.

Today, it is a tangible reality that scientific and technological development provides benefits for a large part of the population in absolute terms. Castells $(1999,2000)$ already indicated two decades ago that access to resources, goods and services was unequal because it was selective. This situation was associated with the constant research for profitability on sustainable social and environmental development. A reflection of this is the asymmetries that we find in the growth between regions, countries, cities or people's vital income. In this process, centrifugal and centripetal movements have been generated that are giving rise to black holes in the world and in cities that have caused cultural environments where perverse connections are produced that are not always visible but must be denounced.

In these disadvantaged areas, there is a recurrent relationship with poor management of public affairs, manifested in weak or deteriorating public service networks and the poverty, functional illiteracy and exploitation that go with them. These environments are the best breeding ground for the consolidation of vicious circles that prevent individuals from joining, maintaining and developing professionally in their socio-labour environments and legal entities from generating a socially and environmentally responsible productive fabric or deploying networks that provide basic life support to citizens in their regions and local communities of reference.

In the educational field Toffler (1990; 1991) also visualized and announced at the end of the last century that technological development would have multiple consequences, thus providing us with great opportunities through interactivity (two-dimensional capacity of response, student-machine and vice versa), mobility (capacity to develop education in any ecosystem which will break the monopoly of classical educational institutions, the School and the University), convertibility (the ability to transmit and process information between different media and networks in order to form complex and multivariate systems of common use), connectivity (connection between education agents with plural sources of information), omnipresence (total democratization of information), and globalization (information without borders or differences). This would be accompanied by access and literacy gaps of different signs due to the appearance of new variables that are characteristic of their continuous mutation.

Among other predictions that have come true, we wish to refer to the fact that the dispersion and decentralization of education has brought us closer to the need to adopt an adhocratic conception of organization when those responsible for education give priority in systems to an increase in bureaucratic tasks, individualism, specialization, standardization, and competitiveness, since this has limited not only the capacity for creativity, but also for developing, applying, and transferring knowledge. This approach is not only reducing the capacity of institutions to fulfil their missions, but also de-professionalizes those directly responsible for education, since the tasks that are entrusted to them are not appropriate to their function and have colonized their working time, which has been to the detriment of quality teaching, research, and educational innovation.

The arguments for and against overlap because the orientation depends on the interests and stakeholders situationally. In the specialized forums, the limitations of the current systems to address the future of a Society where Learning and Continuing Education will be the reference to follow in all areas of our lives are explicitly and implicitly recognized. In any case, whether we like it or not, the transformations will have to be faced irremediably and it will have to be assumed that they are profound since they will not only affect the structures, management, functions, spaces, times and resources but also involve the attitudes and values that impregnate the organizational cultures. Therefore, in any proposal for improvement, knowledge, know-how, and know-how will have to be intimately connected.

The educational reforms that are being implemented in different contexts, and increasingly in shorter periods of time, are the tools that politicians use to favour adaptation to change. The problem that is emerging is that, despite the legitimacy of the procedure, the rules do not seem to be guided by the principles that guide sustainable development for individuals and companies in general and in the university environment. The obsession with intra- and inter-institutional competitiveness, the value given to rankings or protocols imposed by external assessment agencies through verification reports that are generalized in Faculties, Schools, Centers and Research Institutes has forced those responsible for education to standardize processes by detailing the contents in the curricula, competencies, methodologies, times, spaces, resources, relationships or evaluation systems, among other issues, but the procedures followed or the collateral results that have been integrated into the organizational cultures are not questioned. In our opinion, this approach has been exhausting the system and is putting it on the verge of paralysis because it makes it incapable of developing and providing autonomous and flexible responses adapted to the needs, expectations and demands that are generated situationally (Álvarez-Arregui, 2017).

In this regard, it should be pointed out that many natural and legal persons responsible for education seem to have forgotten in practice that their academic, social and occupational responsibility requires them to provide the agents involved with the necessary tools to prepare the new cohorts of professionals and students who enter, transit, graduate and re-enter the system to join a knowledge and lifelong learning economy as responsible citizens who strengthen civil society.

The administrators of public educational institutions also seem to be forgetting that innovation is a continuous process that requires creativity to respond to problematic situations that emerge from the development of multidisciplinary projects based on knowledge where individuals and companies develop ideas, tools, models, protocols... in short, innovations that must be integrated into academic curricula so that universities can implement them, develop them and improve them in their degrees, masters, proprietary degrees and transversal programs of continuous training. When these issues are no longer the object of preferential attention or debate within and outside the university systems, they lead processes, people and institutions towards a mechanical standardization where regulations, routines, competitiveness and bureaucratization end up becoming the rule rather than the exception (Álvarez-Arregui, 2018, 2019).

Educational administrators also seem to have forgotten that in the coming years, in our cultural environment, a large cohort of professionals, including teachers, who began working in the era of expansion (1970-1985) are retiring and will be partially replaced. This situation could lead in the medium and long term 
to a progressive deterioration of many university institutions, but above all of those that have not foreseen, reflected on and acted upon the current selection systems where strong inbreeding and clientelism has been installed, which maintains, if not increases, the precariousness of public services. At present, there is still no desire to intervene in an organizational culture that in higher education has become excessively politicized and revolves around independent or low-functioning cellular groups where intermediate coordination systems have become bureaucratic and where teaching, research and administrative teams can design, innovate, apply or experience excellence or failure independently of the other units or the institution that hosts them (Buckland, 2009). The ambiguity of objectives, the diversity of interests and the struggles for power (Weick, 1967) are perpetuated in many university institutions, slowing down, if not preventing, the development of visions, missions and strategies with a projection into the future, which favours competition and confrontation to the detriment of collaboration, continuous training and sustainable improvement.

Postmodern society is no longer susceptible to incorporating absolute and immovable truths as was the case in modernity, but what is evident is that the person is once again at the center of the process and, like Colom (1997), it seems to us necessary to reorient the mission of education toward knowledge in which humanism is reinterpreted by endorsing a self-sufficient man. In this regard, we consider appropriate the contributions of Toffler $(1990,1991)$ when he stated that education would be learning to innovate, learning to understand change, learning to change and learning to live with change. If his arguments are assumed to be valid, we will have to deploy strategies in the re-interpretation of teaching and of teachers because, by placing learning as a priority focus in the society of the future, the responsibility for education can no longer be in the hands of teachers alone; so joint decisions will have to be taken from political, academic, social and business cultures. The curriculum will also be the object of profound revisions to provide it with a common base where systematic, systemic and continuous innovation will require lifelong training, which guarantees the development, deployment and consolidation of a universal learning curriculum (Álvarez-Arregui, Rodríguez-Martín and others, 2015). Finally, it is necessary to carefully review the results obtained from educational research in order to incorporate them in a circular manner into the system through multiple combinations.

Basically, these changes that we announce as necessary are logical reactions of individuals and companies to adapt to a society in constant evolution. As is usually the case, it has been large companies that have first explored the possibilities offered by the new socio-educational and labour scenario, indicating that those organisations that want to ensure their continuity and existence must promote systems of ongoing training for their workers, where creativity, entrepreneurship, work in multidisciplinary teams, critical thinking, resilience, a positive attitude towards error or social responsibility are the corpus of reference in the curriculum for the development of generic and specific skills. In our case, we consider that intervention in this complex scenario demands the construction of visions of a dynamic ecosystem that integrate political, academic, business and social cultures through multiple relationships that will combine top-down, bottom-up, laterally and transversally.

We are aware that the construction of training ecosystems that combine inclusive training in generic and specific skills will be difficult because teaching, learning and the curriculum are no longer the heritage of any particular institution, but it will be possible if we adopt a systemic perspective in which all agents with responsibility for education participate in a responsible and committed manner. Therefore, it seems necessary to us to present some lessons that we have learned and taught in recent decades about the possibilities and difficulties that accompany change in organizations, since under this approach we will be able to contextualize the evolution that has been followed in the development of the European Higher Education Area and it will allow us to close the article with a proposal for the future that we are currently working on.

\section{Cultural Change: Lessons Learned and Taught from Experience}

We are currently going through a moment that could be described as critical since there are many new scenarios emerging in society, which require individuals and companies to seek answers to adapt continuously in all areas in which they operate, hence the fact that educational R \& D \& i is becoming an inherent part of our personal, professional and social lives in the time we have to live.

The problem of change is its polysemous and it has been associated with other concepts such as improvement, development, reform or renewal, but also with a break (Popkewitz, 1994) with the stable or natural aspects of our social life. Therefore, when talking about change, it should be borne in mind that there are many perspectives that can be adopted to address it and the best decisions are not always made. In our case and in this context, it has seemed appropriate to present some lessons we have learned and taught from the academic, social and business spheres where we are witnesses that Educational $\mathrm{R}+\mathrm{D}+\mathrm{i}$ acts as a sap, an internal energy, which circulates through the public and private system. Some paths have already been adressed, so we will take a brief look at some of those we have travelled in our experience, as we believe that they can serve as guidance, guide or reference for developing visions, missions and strategies from which to explore new paths for a real and widespread implementation of Educational R+D+i.

\section{Lesson I}

Research and practice agree that educational change occurs whether we want it to or not, given that educational organizations, understood as intentional training ecosystems, are in continuous transformation, as is the society of which they are a part, as an effect of globalization; of scientific-technological development; of neoliberalism; and of access to data, information, knowledge and know-how. This situation implies a change of paradigm because it requires us to acquire or develop our competencies through learning, teaching and personal effort since the decisions taken in this regard will have an effect on the axes that guide people's lives (social; work; cultural-leisure; personal; and political) with repercussions of different kinds (Álvarez-Arregui, 2010; Álvarez-Arregui, Rodríguez-Martín, Agudo Prado and Arreguit, 2017; Álvarez-Arregui, Rodríguez-Martín; Madrigal Maldonado, Grossi -Sampedro and Arreguit, 2017).

\section{Lesson II}

The conceptual platforms, models and reforms that are developed from policy go hand in hand with practice, since the change is known once the innovation has taken place and has been assumed by the users. Therefore, the visions based on results have been shown to be insufficient, which is why the focus should be extended to systemic visions where the entry, process and product factors should be taken into account from different dimensions, moments, scenarios and perspectives (Álvarez-Arregui, 2002; Arreguit, 2017). 


\section{Lesson III}

Change in socio-educational ecosystems affects the environment, population, technology and relationship systems, and therefore modifies or influences people, processes, programmes, outcomes, climate and culture. On a personal and professional level, change occurs when a systemic vision is built on education that takes into account the problems that accompany it, the possibilities provided by collaboration, the value of research to generate knowledge, put it to work and improve skills, and the increase in the quality of life of users through learning and teaching shared values (Álvarez-Arregui, 2017).

\section{Lesson IV}

At the organizational level, change is fostered when a shared vision of the whole is built and structures, norms and practices are generated that are consistent with that vision, the capacity to generate professional, institutional, community and knowledge development that makes people, teams and the institution grow in a culture of collaboration in which progress is shared and support (internal and external) is provided in the face of difficulties (Álvarez-Arregui, 2002).

\section{Lesson V}

Change generates uncertainty because macro-trends generate tension, chaos and instability in socio-educational ecosystems, which requires making collegial decisions about organization, curriculum and professional development. Uncertainty is reduced when change is publicly and fundamentally justified (why, what for, what is intended, who is affected, who promotes it...) to different audiences depending on the political, economic, social and cultural moment, they are made participants in the construction of the vision, mission, strategy and implementation and they participate in the impact (Álvarez-Arregui, 2009; ÁlvarezArregui, Rodríguez-Martín, Agudo Prado and Arreguit, 2017).

\section{Lesson VI}

Changes in socio-educational ecosystems must be promoted simultaneously from the top down (reforms), from the bottom up (innovation and organizational-community development), laterally (professionalization, coordination and inter-institutional collaboration) and transversally (projects and multidisciplinary teams) with the intention of learning to learn, learning to change, learning to accept change, learning to manage change and learning to live with change (Álvarez-Arregui, Rodríguez-Martín; Madrigal Maldonado, Grossi -Sampedro and Arreguit, 2017).

\section{Lesson VII}

Collaboration becomes a key reference for addressing change and uncertainty when it is interpreted as a) moral support to bring out the vulnerable aspects by helping to address the failures and frustrations involved in this process; b) increased efficiency, since coordination eliminates duplication and redundancies between the contents being taught; c) greater effectiveness, since the quality of learning is improved by improving the quality of teacher education; (d) mutual support in coping with risks, incorporating a greater diversity of strategies, increasing feedback and generating a climate of trust; (e) reduction of excessive bureaucratic work by sharing burdens and pressures; (f) synchronized time visions by participating in the same perspective on the direction of change based on the existing situation as this gives it greater credibility; g) situational certainty based on the real possibilities for action and existing limitations; h) political assertiveness, since teamwork strengthens confidence in adopting external proposals and the most appropriate moment is expected to consider their implementation, delay it or oppose it if it proves to be dysfunctional; i) increased capacity for reflection as a result of joint work, communication and joint actions; j) improvement of the organization's response capacity, since they are synergic processes resulting from the skills and knowledge that are put in common; $\mathrm{k}$ ) increase in opportunities to learn as a result of the exchange and positive attitude in the classroom, in the department, in the faculty, with families, with students, with other educational ecosystems..; 1) continuous improvement by understanding change as a process that has no end in the research for new solutions to problematic situations that arise (Hargreaves, 1998; Álvarez-Arregui; 2017; Arreguit, 2017).

\section{Lesson VIII}

Change and collaboration are subject to multiple interpretations given that there are many interests and stakeholders around them. This generates deviations of education ecosystems toward complacency, if they are oriented toward safety and avoid conflict; conformism, if it degenerates into a reification of the group that eliminates individual creativity; artificiality, if processes are monopolized by the administration and treated superficially; and co-optionality, when collaboration is used politically to assure the condescension of teachers with education reforms. Therefore, change and collaboration can adopt different paths depending on who controls it, who participates, what its ends are, and what conditions should be established in order to maintain it. Deviations are always latent because they are intrinsic to any process of adaptation, hence the need to articulate situational intervention proposals based on ethical behaviours shared by the people involved, and where the direction and style of leadership will play a fundamental role in their promotion, defence, development, implementation, review and sustainability (Hargreaves, 1989; Álvarez-Arregui, Rodríguez-Martín, Agudo Prado and Arreguit, 2017).

\section{Lesson IX}

The causes that make learning and entrepreneurship difficult in organizations are due to multiple factors. In some cases, they are endogenous, in others exogenous and in most cases mixed and linked to the organizational cultures that have been consolidated. The last one seems to be the most appropriate given the interdependence generated between some socio-educational institutions that develop situationally and circumstantially and to the demands that are generated in a globalized society, with a high technological development, with an unlimited capacity of access to data, information and knowledge, with some social networks that impregnate all the areas of our lives and with a hegemonic neoliberalism that conditions the social and educational policies and, therefore, the projects in the institutions (Álvarez-Arregui, 2002; Arreguit, 2018).

\section{Lesson $X$}

Change requires facilitators: motivation (availability, interaction environment, reinforcement of teamwork, animation of dialogue...); justification (remembering the purpose of change and periodically reinforcing the commitment by relating the 
process to the usual tasks...); political support (locating those external-internal agents that can block or distort the change process by establishing contacts to improve relations, blocking resistance, seeking their agreement...); and sustainability (managing change by providing resources, incentives, training, advice, visibility...). In addition, decisions must be made about the dilemmas that emerge in the process (individual, team, departmental, institutional, community, structural, organizational...) (Álvarez-Arregui, 2017).

\section{Lesson XI}

Change is accompanied by strategies of different kinds: coercive (determined by the administration), rational (confident that its design will lead to acceptance, internalization and implementation) or re-educational (aimed at achieving the commitment of the people involved to support the improvement by adapting the context and ways of thinking and doing). They can be applied in the different phases of change: initiation (awareness, dissemination and debate of knowledge); development (schoolbased review); and institutionalization (evaluation of the change process) (Bolivar, 1999; Álvarez-Arregui, 2017).

\section{Lesson XII}

Change must be sustainable, hence several keys associated with improvement must be considered if we are to go beyond the processes of schooling. Thus, it must endure, it must be supported by sufficient available or achievable resources, it must not harm other institutions and systems, and it must promote diversity and ecological capacity throughout the educational and community environment (Fullan, 1991; Álvarez-Arregui, Rodríguez-Martín; Madrigal Maldonado, Grossi-Sampedro and Arreguit, 2017).

\section{Lesson XIII}

Sustainability is strengthened when different local cultures are integrated into the reform process so that they can be reinterpreted situationally from a constructive dialogue that favours diversity, something that goes against the current reforms that are being promoted at the international level, where standardised comparison when imposed as a norm increases inequality (Hargreaves and Fink, 2002; Álvarez-Arregui, 2017).

\section{Lesson XIII}

Change and leadership are associated, hence those people who want to promote them with projects, who occupy positions of responsibility by appointment, or who have been chosen by the organization for their management must know the empirical evidence and good practices that support the improvement processes that have taken place or are being developed in other organizations in the same field. Communication, delegation, promotion of innovation and humility are basic references in the management of organizations and R\&D ecosystems (Arreguit, 2018).

\section{Lesson XIV}

The review of empirical studies and from the practice itself does not indicate that WE cannot command what should be done; change is a journey; problems are our friends; the vision is built among all people; individualism and collectivism have equal power; neither centralization nor decentralization work in isolation; anyone can become an agent of change; internal organizational cultures sift external prescriptions; relations with the environment can be critical; linear approaches are insufficient; and the construction of valid organizational knowledge is complex because it has to be able to relate, contextualize and globalize sequentially and simultaneously at different levels (Fullan, 1991; Álvarez-Arregui, 2017).

\section{Lesson XIV}

Socio-educational institutions have progressively reduced their learning capacity because the institutional documents they use to guide their development have not crystallized as references and guides for intervention strategies. The bureaucratic orientation given to them by those responsible for institutions exercising transactional leadership has encouraged, explicitly or implicitly, by standardized criteria that have been supported by those educational administrations that prefer control and dependence to development and continuous improvement and that these organizations are managed under criteria of greater autonomy and participation of the educational community (Álvarez-Arregui, 2002).

\section{Lesson XVI}

It is not perceived that there has been an intention of real educational improvement on the part of many institutional promoters, external and internal, to rely on institutional documents that gather shared visions, missions and strategies in order to project their communities of reference towards the construction of a strong culture of inter and institutional collaboration where the interests, demands, needs and expectations of the different sectors are reflected, for which reason there has been no identification with the principles that guide them and they have ended up being generalized as administrative documents (Álvarez-Arregui, 2007).

\section{Lesson XVII}

The paradox that we have found in those institutions with more developed organizational proposals based on comprehensive approaches, with projects underway at all stages, with top-down and bottom-up proposals, with high levels of participation and with a more pedagogical, transformational and professional direction, is that they do not consider that their Institutional Project and/or Strategic Plan is complete, since they have interpreted it as the result of a dynamic process in continuous development, so they use different feedback systems to constantly improve them (Álvarez-Arregui, 2017).

\section{Lesson XVIII}

The learning of legal entities, organizations, has been negatively affected when management teams and intermediate coordination systems have not assumed their institutional and social responsibility in the development of shared projects and have preferred to opt for restrictive organizational models where hierarchical proposals prevail over horizontal ones, where some collectives emerge and others are relegated, where single-person and collegiate bodies are used as a mechanism for the formal legitimisation of institutional policies and where reflection, constructive debate, intervention planning, process deployment and decision-making based on maintenance-oriented bureaucratic reviews have been hijacked (Álvarez-Arregui, 2017; Álvarez-Arregui, Rodríguez-Martín, Agudo Prado and Arreguit, 2017). 


\section{Lesson XIX}

Learning and teaching in organizations suffers when apathy towards planning is increased by the routine processing of institutional documents whose main objective is to justify themselves to educational administrations. This situation is not reached by chance but by the concurrence of situations and processes that are consolidated in time, protected by a formal, academic and homogeneous planning that is legitimized in the institutional symbolic rites, knowing the existing incongruities (Álvarez-Arregui, 2017).

\section{Lesson $\mathrm{XX}$}

Common weaknesses in the way management or institutional leaders approach change have been identified in organizations that have difficulty in learning and teaching. In this regard, it should be borne in mind that when organizations are to be led, proposals for the future should not be made if the necessary conditions are not met, since this situation will end up turning against the promoters if there is a dissonance between what is said to be done, what is actually done and what can be done (Álvarez-Arregui, Rodríguez-Martín; Madrigal Maldonado, Grossi -Sampedro and Arreguit, 2017).

\section{Lesson XXI}

People who want to assume institutional responsibility must be aware that there are many attitudes that can be adopted to the views of the organization, hence it is relevant to communicate information as objectively as possible, which is not usually the case when it is peppered with preconceived opinions, personal biases and ideology. In presenting the desirable future for the institution, it should be borne in mind that a) true commitment is rare, managers want workers to participate in the vision, but this approach is based on transactional leadership b) enlistment leaves elections free and, although the vision is supported, it does not yet feel like a partner, so spaces must be opened up to share it, incorporating other contributions and making them participants in it; c) in the face of disobedience and apathy, in some cases it will be necessary to try to listen to the reasons and in others to try to create hope, but it will be necessary to act situationally according to the circumstances, reflecting on the why, but being aware that the best thing is to avoid hypocrisy or to generate false expectations that in the long run derive in confrontations, hidden blackmail and open conflicts; and d) when one starts off or adopts excluding starting positions, little can be done (Senge, 1990; Âlvarez-Arregui, 2017).

\section{Lesson XXII}

The output of the reforms varies according to the degree of dissemination of their goals, of the strategies and of the methodology on which they are based, but, above all, they should have broad social support, promote the greatest possible political consensus, be considered in the long term, raise the awareness of those involved, and avoid organizational and curricular impositions. These determining factors cannot be dissociated from the regulatory role that public administrations and national and international bodies have, but by establishing a distance from partisan curricular policies we consider it necessary to support the initiatives of schools, to ensure that educational inequalities are not promoted and to support a public, democratic, transparent, inclusive, creative, entrepreneurial and innovative educational system (Álvarez-Arregui, 2017).

\section{Lesson XXIII}

In the process of change, attention must be paid to institutional leadership, the attitude of teachers, the involvement of the educational community and the support of the local political-institutional environment, since their role is not only relevant but also decisive. The prominence must extend to all people and support with greater intensity the core of operations because it is in the direct contact between teachers, professionals, students and families where more tensions or greater satisfaction are produced. The change must generate a system of continuous learning that is incorporated into the organizational culture under the principles that inspire inclusive organizations that learn and undertake (Álvarez-Arregui, Rodríguez-Martín, Agudo Prado and Arreguit, 2017).

\section{Lesson XXIV}

An inclusive organizational culture must and can be learned, because it is acquired and transmitted by observation, experience and study; it must and can be shared, because it affects all people as well as their personal, social and professional development; it must and can change perceptions about education and the problematic situations that accompany it; it must and can have the capacity to adapt, since it is based on the evolutionary and adaptive capacity of the human being; It must and can be transgenerational, since it accumulates and passes from one cohort to another; it must and can be inter-institutional, because good practices can be exported; it must and can be global when inclusive education is extended to localities and cities through comprehensive policies and from social networks; and it must and can be projective if it is focused on the future with the intention of improvement (Álvarez-Arregui, 2002; Tonucci; 2009 Booth and Aiscow, 2015).

\section{Lesson XXV}

The errors we have found most commonly when considering changes in organizations are psychological error (emphasis is placed on individuals without taking into account the relationships of roles, norms and values of the organization that make up the system of which they are a part); sociological error (it is thought that changes in structure will result in a change in the behaviour of members and that this change will occur in the planned sense); rationalist error (it is believed that talking to people about the convenience of change will occur and there is a tendency to assume that a rationally justified change will be in the best interests of an institution and will be adopted automatically.); temporal error (occurs when the change is not considered at the right time, given that there are other causes that concern people more, be they labour problems, internal conflicts, excessive bureaucratization, other concurrent changes simultaneously); and cultural error (is detected when it is not taken into account that in organizations there are different subcultures that must be managed). When we do not act on the cultures of the organizations, they end up imposing themselves on each other in a process of hidden cultural colonization from which it is impossible to establish solid references to develop a shared project that responds to the type of institution that we want to promote; the style of leadership that we want to promote; the type of professional relations and with the environment that we want to deploy; the learning and teaching methodologies that are to be implemented; the conditions under which action is to be taken; the degree of institutional autonomy that is desired, 
needed and to be used; the measures to be adopted to develop inclusive education; and the social responsibility assumed towards the environment under the objectives for sustainable development that guide Agenda 2030). (Álvarez-Arregui, 2002, 2017; Álvarez-Arregui, Rodríguez-Martín, Agudo Prado and Arreguit, 2017).

These and other lessons, which have been learnt and taught in recent years, have not been sufficiently taken into account in the construction of the European Higher Education Area, hence it has had and must have been the subject of constant revisions that have focused on design and implementation. In the coming years, much more emphasis should be placed on learning and teaching common shared values, which entails an important process of reculturation in all areas and levels of responsibility of this socio-political, legal, economic, labour, technological, leisure, cultural and sustainable ecosystem.

\section{An Ecosystem of Learning in our Cultural Environment: Higher Education in Europe}

The present moment, although at first sight it might be described as complex and uncertain, may be propitious to initiate processes in university institutions that find it difficult to build their visions and develop strategies that allow them to adapt to continuous change more effectively and to face the challenges of the future with greater guarantees of success. Those responsible for education are aware that in the current format these organizations are committed to promoting internal restructuring and reculturation processes, if they do not want their consolidated missions, associated with teaching and research, to lose prominence and be displaced as educational references in future societies.
Universities are seeing that widespread access to technology, data, information, knowledge and know-how is imposing continuous and systemic innovations that affect people's skills (knowledge, know-how and expertise), whether physical or legal, so that the use of intensive, accessible, versatile and lowcost platforms for management, research, teaching and transfer are becoming progressively more relevant because of the added value they generate for processes and results. This exponential evolution of technology and knowledge leads inexorably to a dynamic society where most students will practice professions and use tools that have not yet been created. Faced with this situation, curricula, learning models, systems for selecting administrative and service staff, teaching and research staff, competence assessment, standardisation, certification and bureaucratisation as we know them must evolve (ÁlvarezArregui, 2018). The task will not be simple because there are many dimensions and variables that have been intertwined and emerging in a finite physical environment derived from the concurrence of neoliberalism (Bell, 1991; Gimeno Sacristán, 2001; Pérez Gómez, 1998), globalization (Castells, 1999; Santos Guerra, 2000; Gairín, 1996), the scientific-technological development (Dosi, Friedman, Richard, Silverberg and Soete, 1990) and an increasingly open access to data, information, knowledge, wisdom, learning, teaching and innovation (Drucker, 1969; Böhme and Stehr, 1986; Morin, 2001; Bolívar, 2000; ÁlvarezArregui, 2017; Xavier 2018).

The described framework, (see the chart), has direct and indirect recurrences in the ecosystems in which we are integrated - social, labour, leisure, cultural, personal and political - acting as a reference, guide and support because they are constructs that constantly feedback.

\section{ENVIRONMENT PHYSICAL - FINITE}

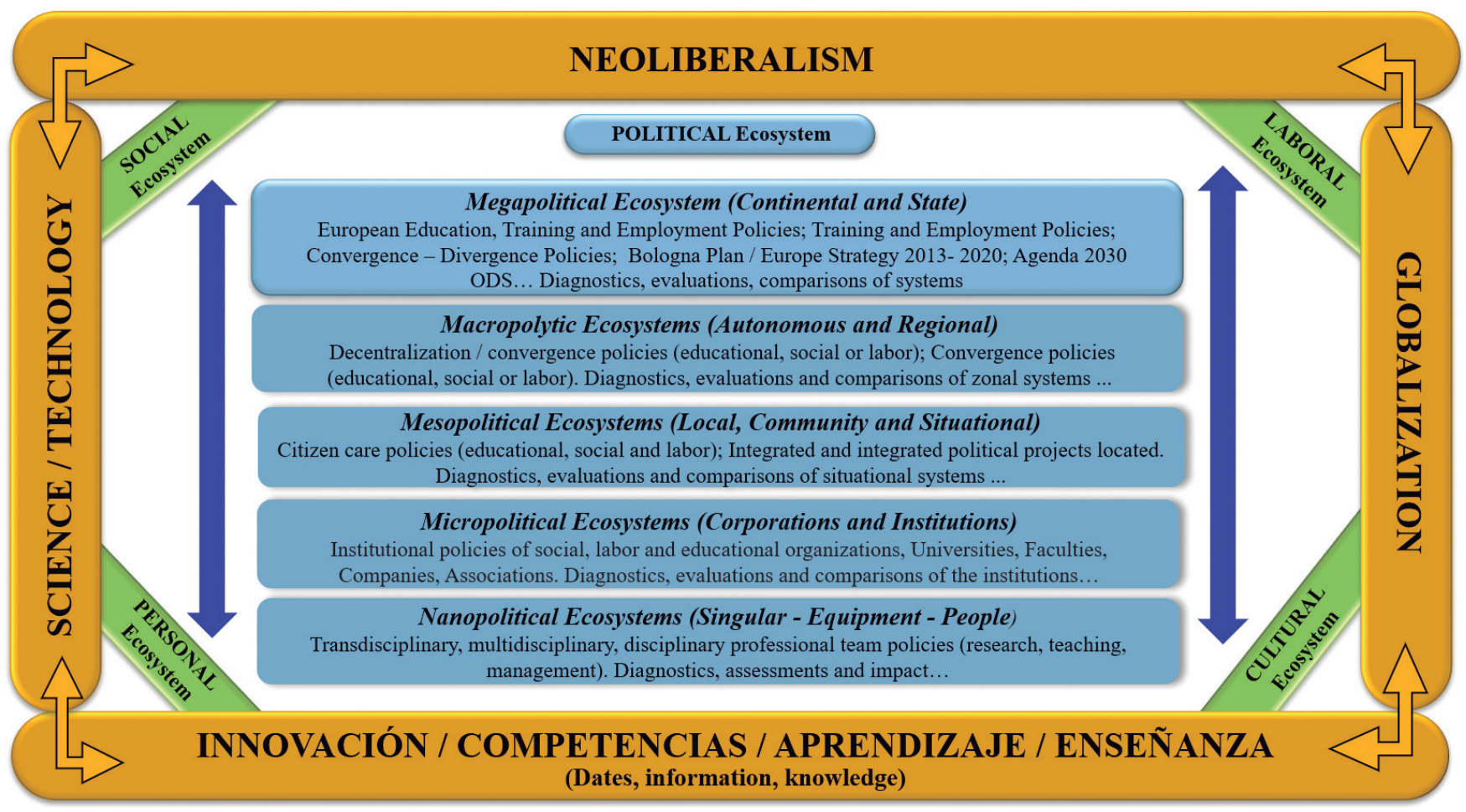

Chart 1. Global ecosystem vision and exemplification of political ecosystems in dynamic interaction 
The problem we are facing is that macro-trends interact from what are called "non-linear dynamics" and, due to the exponential evolution of technological development, a higher acceleration is caused in all the competing ecosystems, which forces individuals and companies to a continuous innovation from a lifelong learning and education. In this respect, universities should be reminded that, although they have been places of knowledge generation and transmission in recent centuries, they must reflect, understand and take decisions on their current situation with regard to knowledge transfer, on the use they make of technology, on their capacity to anticipate future situations and on the added value generated by their knowledge transfer processes in order to provide viable solutions for sustainable socio-economic, political, legal and educational development in their regions of reference in particular and in society in general. Therefore, the great challenge we face will be to enable individuals and corporations to survive in a supranational reality of blurred boundaries by constantly rebalancing the global with the state, the local with the institutional and the personal with the public and private. This scenario makes it necessary to carry out precise diagnoses that allow for the establishment of agreements, ways of collaboration and explicit commitments between all parties. This is why we continue to review some interesting initiatives that have been proposed and others that we are working on, given that the construction of university ecosystems that combine inclusive professional development in generic and specific skills can no longer be considered in isolation, because teaching, learning, curriculum or transfer is no longer the heritage of any particular institution. Therefore, the intervention must adopt a systemic approach in which all actors with responsibility for education participate in a responsible and committed manner.

The model that has been deployed as valid in our cultural environment is the European Higher Education Area (EHEA), its development has been implemented in recent decades in different countries through some axes that are adapted situationally and from which it is intended to address the current challenges and future of Higher Education. It is therefore necessary to take this framework into account as a starting point for any model that one wishes to promote because it integrates the basic principles to be followed by university institutions and therefore impregnates, to a greater or lesser extent, the organizational and functional visions that are deployed in these institutions. As a reminder, they are presented schematically:

\section{The first axis raises the need for training in and by competencies}

The literature has been very prolific and controversial on this point, and currents of opinion are swinging from uncritical to irreconcilable positions between the competency-based approach and university pretensions (Carabaña, 2011). On other occasions it has been research, analysis and conceptualisation that have guided its development, orienting it towards the labour market (Alonso, Fernández and Nyssen, 2009). Be that as it may, the axis has been incorporated to the regulations of the different community countries which has generated a modification of the planning of the formation programs that has been arduous, because in their design and implementation the competences have been understood as "complex structures of processes that people put in action-action-creation to solve problems and to carry out activities of the daily life and of the labour-professional context, oriented to the construction and transformation of reality" (Ruiz Corbella, 2006: 100).

In the face of such an open approach to competencies, it is logical that the task remains unfinished because it goes beyond the educational (concepts and procedures, academic knowledge) and adopts a transversal educational dimension in which axiological and attitudinal issues (knowing how to be) are integrated together with knowledge and know-how. This approach, already pointed out by Delors (1996), has been described as inappropriate for higher education by some academics who indicate that they devalue knowledge; something in which those of us who support the added value derived from education by the multidisciplinary component it incorporates into research, teaching and innovation disagree deeply. Furthermore, we consider that academic freedom is not altered since it leaves room for developing the intangible intellectual assets that the university tradition has always cultivated, although we disagree when it comes to restricting the intrinsic potential of competition from measurement, quantification and standardization. In any case, we support those models that prioritize learning over teaching (Álvarez-Arregui, 2017; Gairín, 2003; González and Wagenaar, 2003; Rué, 2004) since they give greater prominence to the individual and situate the teacher as a guide and facilitator of a process aimed at achieving clearly defined objectives. Furthermore, it is more permeable to work in multidisciplinary teams for the development of projects and revitalizes tutoring, because it goes beyond the mere resolution of difficulties derived from the programs, which requires initial, continuous, transversal and on-demand training according to the needs and expectations that are raised.

\section{The second axis refers to professionalization and employability}

The education of professionals has always been one of the functions of the university, but in today's learning and teaching society the change of perspective is evident if one wants to put the emphasis on the type of professionals one wants to train. In this respect we have been sharing those initiatives that present the EEES as "a good opportunity to introduce topics of civic and moral development in the new curricula to train the future professionals of our society, who will be able to act in a responsible, free and committed way" (Boni and Lozano, 2006: 11). Despite the good intentions that underlie these arguments, we believe that they are far from being generalized. The palpable consequence is a progressive distancing from the authentic ideal of education where the percentages of professionally prepared specialized graduates are not so relevant, but rather the attitudes and values (knowing how to be). This fact limits the political will contained in Royal Decree 1393/2007 where it was stated that "the new organization of teaching will increase the employability of the graduates (...)". At this point, we believe that there is still a disconnect between the university and the work environment, which should be reinforced with new bridges of understanding, as well as a review of the concept of employability, since over time it has become a desideratum because the market is not capable of absorbing the number of graduates that each discipline provides, which generates tensions between university education and employment (Santos Rego, 2014). In any case, these imbalances that are detected between training and employability cannot result in a lack of recognition of the intrinsic value of knowledge, but rather it becomes necessary to reconstruct the model by placing more emphasis on Dual University Training, which again endorses greater involvement of politicians, social entities, companies, academics and citizens (Álvarez-Arregui, 2017, 2018, 2019).

\section{The third axis concerns research}

The production of knowledge that characterises another of the University's missions has been shifting towards high specialisation, 
is eminently applied and is being oriented towards transfer and innovation with more positive repercussions for large companies than for medium and small enterprises. This point should be the object of special attention in the coming years because, as Edgar Morin (2009: 44) indicated, in the situation we have reached, hyperspecialisation prevents us from seeing the global, the whole, in such a way that with this approach we pay attention to what is essential and indispensable, knowledge is compartmentalised and dissolved from the disciplinary specialisation. The problem is found in practice, since situations that require solutions are never fragmentary, so multidisciplinary approaches are required to address the objects of study in the current context. The European Research Area took this deficit into account by "establishing a research area without frontiers in which scientific resources are better used to promote employment and competitiveness in Europe (...) by combining three concepts: the creation of an internal market for research, restructuring European research, establishing a European research policy" (Commission of the European Communities, 2000). Although there has been progress in this direction since the EHEA, decisions are being made that may have undesirable effects on the development of natural and legal persons if the mistake is made of bureaucratising scientific results or if priority is given to private initiatives backed by large corporations to the detriment of the findings of those disciplines that offer keys of value to human beings.

Despite the fact that strategies for the development of the Arts, the Humanities and the Social and Legal Sciences are being targeted, we continue to find greater resistance from these areas of knowledge to the process of convergence of the EHEA than from the natural sciences and technical fields (Pechar and Pellert, 2004). In any case, the internationalisation of research and the results that derive from it is, in the words of Scott (2006), a mission of the university that serves the nation-states, so it cannot be considered to be dissociated from teaching and the value it brings to national public service. This approach once again endorses the need to develop multidisciplinary research projects that require forging alliances between politicians, social entities, companies, academics and citizens in the development of research projects.

\section{The fourth axis is associated with internationalization and mobility}

The systemic and glocal perspective is once again present in this axis, as value is placed on the international relations established between institutions, teachers, students and professionals (Tennant, McMullen and Kaczynski, 2010: 6), which supports an open curriculum with a strong international/universal base, since this approach provides a more generic range of skills that favours the professional profile of people, thus increasing their employability, but also is likely to integrate the culture of the native country and project it abroad and vice versa, thus deepening the feelings of community membership and integration. In any case, we must be alert to possible deviations, because it may be used as a strategy of competition in the global market to attract international human resources, which could distort the initial claims. The capacity of universities to host international groups and offer training programmes that stimulate the intercultural experience should also be reflected upon, deepening the socio-educational, academic and research dimensions from a European and global perspective. Finally, it will be necessary to recognize that homogenization has taken precedence over harmonization, so that curricular plurality has suffered as an effect of standardization in such a way that the single model continues to run the risk of not being assumed in order to develop the competencies that will be needed in the European labour space and can also lead to an increase in frictions with university traditions in which liberal training, universal education and wisdom have always had a representative place (Barnett, 2005).

\section{The fifth axis is linked to the socio-educational dimension and European citizenship}

The Bologna Declaration (1999) called for a deepening of Europe's social and cultural heritage, so it made a commitment to globalisation, scientific and technological development, market conditions associated with neoliberalism, shared access to data, information and knowledge, which revitalised lifelong learning as a fundamental cross-cutting issue in the EHEA. In this context, different projects have been deployed such as the Jean Monet Chair to prevent shared common values from overcoming a reductionist conception of a concept of citizenship associated with consumption, performance or the mercantile treatment of knowledge from civil society. At this point it is important to emphasize once again the value of glocalization, since this metaphor allows for transfers between local and global identities, expectations, demands and needs, favouring the construction of a European citizenship where people see their unique roots reflected, but at the same time cosmopolitan where diversity and common cores of meaning lead us towards an authentic civic education. In short, we share with López-Gómez (2015) that the substantive nucleus of values and purposes that Europe has assumed as a tradition for the development of the EHEA must be a common substratum of deep draft if it is not to succumb to a mercantile or ideological utilitarianism.

\section{The sixth axis considers competitiveness and quality assurance}

Competitiveness and quality have been two fundamental pillars in the process of European convergence. The Salamanca Communication (2001) already indicated that quality is not enough to be given, it must be demonstrated and guaranteed so that it is recognised and appreciated by students, decision-makers and all of society in the country, in Europe and in the world. That is why expressions such as accountability, stakeholder, quality assurance and performativity have been occupying a representative space in EHEA initiatives. Tennant, McMullen and Kaczynski (2010: 8) reminded us that higher education has increasingly been a resource that must be organized to maximize its contribution to economic development. This fact is important because the demands on individuals and legal entities involved in higher education make demands on universities based on the public investments made in them, hence the incorporation of expressions such as accountability in political, academic and social cultures where transparency in the new model of Public Management is gaining new momentum.

In the case of Spain, ANECA and other autonomous community agencies carry out assessment, certification and accreditation of teaching staff, proposals for study plans in the EHEA, monitoring and accreditation of official qualifications, teaching, internal quality assurance systems for qualifications, and assessment of awards for excellence in doctoral studies, among other tasks. These processes and actions aim to reflect the effectiveness of practices by evaluating what is intended to be measured with identified standards that make the university concerned about what works and can be demonstrated (Pasias and Roussakis, 2012: 317). At this point we must again be attentive to the deviations generated under this approach since, as Power (1999) points out, the institutionalization of large-scale checks is giving way to perverse bureaucratic systems that are causing the foundations of support 
to crumble in the face of constant demands for information, for the allocation of resources to surveillance or for the exhaustion and demotivation of human capital to carry out their teaching and research functions under conditions of latent and present control. It seems then that, despite the security that evidence and quality evaluation can provide, it is necessary to recover the value of culture, initiative and values that have accompanied the university institution to build an EHEA.

In our opinion, competitiveness and quality are insufficient to build a 21st century university that aspires to integrate and be a protagonist in the process of change that accompanies the transition to a global learning, teaching and knowledge society. This purpose requires learning, teaching and continuous training based on the development of common projects in which representatives of academic, political, social and business cultures participate in a responsible manner. That is why, if we want to make a firm commitment to a pattern of sustainable socioeconomic growth, it must be solidly based and, to this end, it is necessary to create innovation, entrepreneurial and creative ecosystems, with a short, medium and long-term vision, with sectoral coordination, from interinstitutional cooperation, with sustained investment with greater public-private collaboration and with a public commitment among all the agents involved. Academia, business, public administration, politicians and social agents cannot act in isolation and must develop strategies for shared intervention if their objective is to develop sustainable educational innovation based on a continuous transfer of knowledge, know-how and know-how between business, academic and social science and technology systems and vice versa.

In our opinion, any training/learning ecosystem that wants to be oriented towards the acquisition and improvement of skills for all people must be oriented at least from the standpoint of rationality, flexibility, permeability, collegiality, professionalism, multi-disciplinarily, self-management, creativity, inclusiveness, transferability and complexity. This approach involves simultaneously adopting a global, local, institutional and personal approach (Álvarez-Arregui and Rodríguez-Martín, $2013,2014,2015$ ) and distances itself from those disciplinary visions of the curriculum, because it goes beyond the isolated consideration of people, objectives, contents, evaluation or experiences and integrates added value thanks to the Internet of things, automation processes, Big Data, process simulation or the values that are impregnating the global environment such as those emanating from Agenda 2030.

Entrepreneurial learning and teaching ecosystems thus become a viable alternative for responding to the demands of a complex society, since they address those issues that are being given great importance in the socio-educational, economic, cultural, leisure, political, trade union and labour environments. In this respect, the skills to manage one's own learning and teaching; to have elements and methodologies to develop flexible teaching-learning processes; to communicate and share knowledge without linguistic and cultural barriers; to enrich learning and teaching with the participation of professionals from companies, administrations, NGOs, etc. and, finally, to use the presential and virtual spaces according to the needs to provide flexibility to the model and to favour the construction of learning and teaching ecosystems that respond to the demands of personal, professional, institutional and community competences.

\section{Basic foundations of a proposal for change with learning and teaching ecosystems}

In recent years, we have been working on the development of an eco-systemic training model (Álvarez-Arregui, 2010; Álvarez-
Arregui and Rodríguez-Martín, 2015; Álvarez-Arregui, RodríguezMartín, Maldonado, Grossi and Arreguit, 2017; Álvarez-Arregui, Rodríguez-Martín, Agudo Prado and Arreguit, 2017) that is sustainable, open, flexible and inclusive, and that meets the needs, expectations and demands of people, institutions and the environment. It is our goal to overcome the limitations posed by other more rigid, reductionist, bureaucratic and mechanistic approaches in their conceptualization and implementation.

Our proposal takes into account other models such as the Knowledge Ecosystem (Shrivastava, 1998); the e-learning ecosystem based on technologies and systems for managing and supporting learning (Ismail, 2001); the Modular Ecosystem (Dimitrov, 2001); the e-learning ecosystem for work and learning (Wilkinson, 2002); the e-learning ecosystem for professionals (Brodo, 2006), and the Learning-Ecosystem by Gült and Chang (2009), among others. These models incorporate, among other components, a learning design, a population (teachers, students, educational agents, etc.) and a training system for the development of basic skills and web 2.0, 3.0 and 4.0 applications. In this context we are aware that a model can be designed from different approaches depending on the perspective adopted, since there are many parameters, coordinates, factors and variables that concur situationally as discussed in previous references. That is why we present in a synthetic way those fundamental principles that have been guiding our work in recent years (Álvarez-Arregui and Rodríguez-Martín, 2014, 2015, 2016; Álvarez-Arregui, Rodríguez-Martín; Madrigal Maldonado, Grossi -Sampedro and Arreguit, 2017).

The latent danger that we detect in these proposals is that they may end up sliding, perhaps in excess, towards e-learning alone (Uden, Wangsa and Damiani, 2007), for which reason it seems more advisable to us to situate ourselves in a mixed situational learning (blended situational learning) to take advantage of the potentialities that continuous technological development offers us to generate spaces of learning that are present and virtual and that integrate the different concurrent groups, covering all possible possibilities in the face and virtual space (top-down, bottom-up, laterally and transversally), incorporating systems (for tutoring, advice, self-management of learning, management of data, information, knowledge and know-how...), using own and third-party resource bases where we learn to do, learn to innovate, learn to learn, learn to transfer and learn to be by sharing values (Álvarez-Arregui, 2017; Álvarez-Arregui, Rodríguez-Martín, Agudo Prado and Arreguit, 2017).

A shared vision and values. In an EAECE (Ecosystem of Creative and Entrepreneurial Learning and Teaching) a vision has to be agreed and shared in such a way that it integrates the different subcultures in which the different professionals participate and that it is coherent with common objectives that are beneficial for all people.

Distributed leadership. In an EAECE the different professionals and people involved must have opportunities for personal and professional development in such a way that their leadership and self-management capacity is strengthened, as well as their capacity to work in multidisciplinary teams, to develop creative projects, to communicate initiatives aimed at improving learning and to better perform the functions assigned to their job. Leadership should be situational, combining educational, transformational, delegated, inclusive, sustainable and with institutional and community projection.

Individual, collective, organizational and open learning. An EAECE should identify the potential of individuals, teams and the organisation to generate synergies within and outside the institution. It is important to detect what they need, what 
they want to learn, what they need to be trained in and how they should do it to generate knowledge, manage it and share it. This approach drives the ecosystem, because it makes it grow through the construction of a multidisciplinary socioprofessional learning network, where trust and continuous improvement revitalize the organization's capacity to diagnose, plan, implement and learn to undertake from continuous training, creativity, problem solving, project development, team building and strengthening of socio-emotional networks.

A process orientation towards sustainable development in an EAECE, professional practice ceases to be private and becomes public. Observations, records, discussion groups, forums, institutional documents, protocols and previous experience will be some elements to be used for sharing, reflection and improvement of practice, ensuring that learning is pragmatic, collaborative, motivating, open and transformative.

A promotion of trust, respect, negotiation and mutual support as the basis of relationship systems. In an EAECE all people should feel supported, valued and integrated, because when this happens, trust is generated, and commitments are made to the projects and improvement processes promoted. Individual differences, dissent and error are accepted within a critical reflection that promotes the development of the team, and there is no dichotomy between individual and collective.

Outreach through inter-agency networks and partnerships to share and exchange information and knowledge. In an EAECE, interdisciplinarity, interdisciplinarity and trans-disciplinarily are favoured, promoting systems of relations that transcend the limits of the teams, the projects and the organization. Disagreements are negotiated, links are built, and solid links are established between the teams, between the teams and the institution, and from all of them with the environments in which they are projected and with which they collaborate. In this scenario, the ecosystems grow and develop through professional, personal and institutional networks, resulting in open spaces where information and communication flow and learning and knowledge are generated, which has an impact on the growth of people, teams and the institution throughout the innovation process.

Commitment and a social responsibility with the environment. In an EAECE, commitments are made to improve the teams, the institution and the environment. It is valued that risks are taken when initiatives are promoted and the fear of criticism of mistakes is diluted, since they become a source of learning. Support is invited when social and labour problems arise and it is guaranteed that personal, professional, institutional and community successes are shared, as well as the transfer of improvements to the local environment and to society in general.

Collaboration as an organizational, functional and value-learning pillar. In an EAEC certain elements of physical, structural, economic and emotional order are guaranteed so that spatialtemporal conditions of collaboration and shared learning are ensured. In this direction, resources such as work materials, information, advice, multimedia material, protocols or other elements determined as necessary to favour learning and teaching in the ecosystem should be provided.

Ultimately, an EAECE is constantly refined in its different phases, see figure, through diagnosis, creation, planning, implementation, evaluation, transfer, and impact research. In this process, the EAECE can discover new ideas, to integrate them and to transform them towards its objectives. It therefore has the capacity to learn and teach how to learn, to learn and teach how to improve, to learn and teach how to adapt and to learn and teach how to grow in a sustainable way.

Taking into account the above-mentioned references, we continue to work on the development of a university learning - teaching ecosystem which, as a platform for the acceleration of educational innovation, is being integrated transversally into the educational, social and labour fields of the University of Oviedo through innovation, research and educational transfer projects. The administrative form it has adopted is that of the Educational $\mathrm{R}+\mathrm{D}+\mathrm{i}$ Institute (INIE), which is constantly evolving to adapt to the different situations that are emerging at the University of Oviedo.

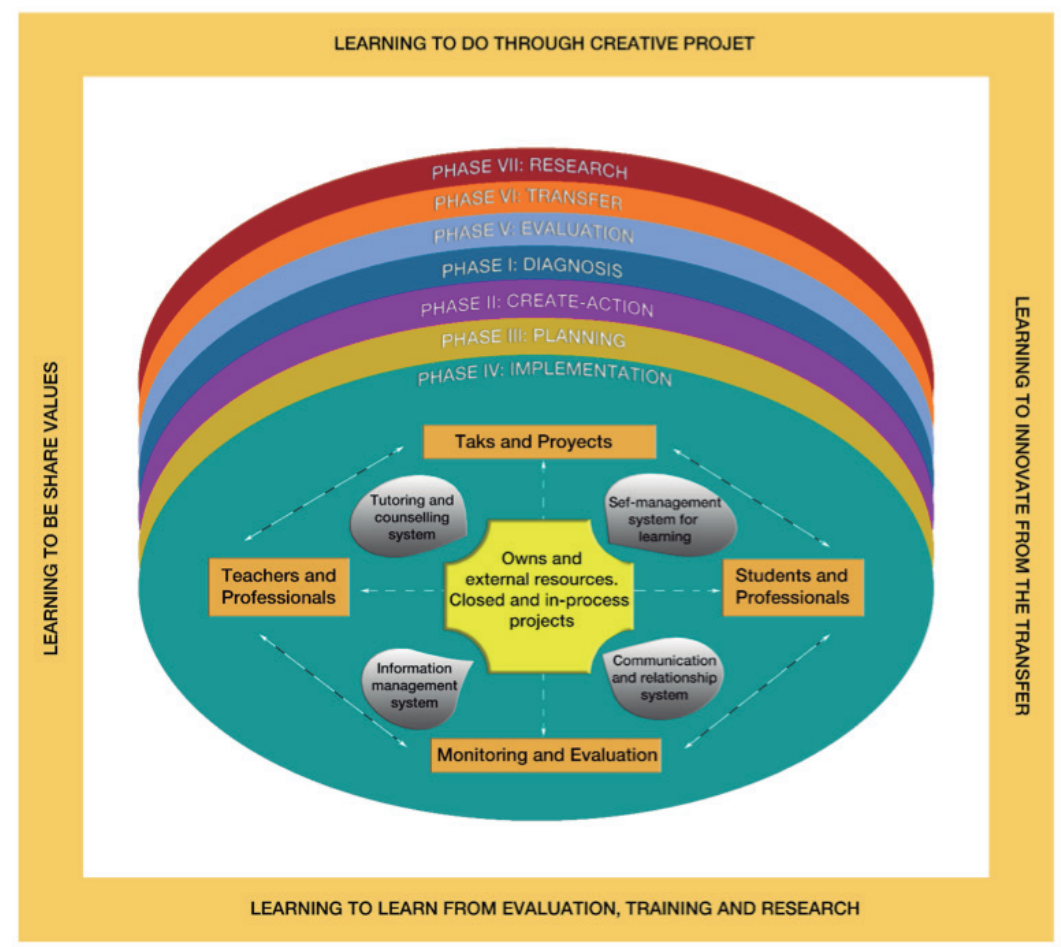

Chart 2. EAEC: Framework, phases and references. Creative and entrepreneurial learning-teaching ecosystem. 
This learning and teaching eco-environment integrate in its design, structure and operation the principles and processes presented that we want to convey to the people and organizations with which we interact. Another important feature is its adaptability, since it can be replicated in different contexts depending on the demands, expectations and needs of the organizations, institutions or teams with which we interact. Being consistent with our vision, we give great importance to the initial analysis since it must capture the complexity of the components of the system and assume the need to integrate the social, educational, labour and political dimensions of the competing components in any intervention project. In any case, the starting point is based on a diagnosis of the available resources in terms of physical elements (facilities, tools, knowledge, know-how...), human elements (profiles of teachers, students, professionals, advisors, users...), financing, available resources, visions, strategies, expectations of the promoters and attitudes of trust and collaboration necessary to integrate academic, political, social and business cultures. From here, a vision is created from a dialogical approach in which one learns to learn, to collaborate, to undertake and to accept in change.

The mission, strategic planning, collaboration and continuous evaluation guarantee the transparency of the processes and make it possible to articulate procedures to regularly review and restructure the vision.

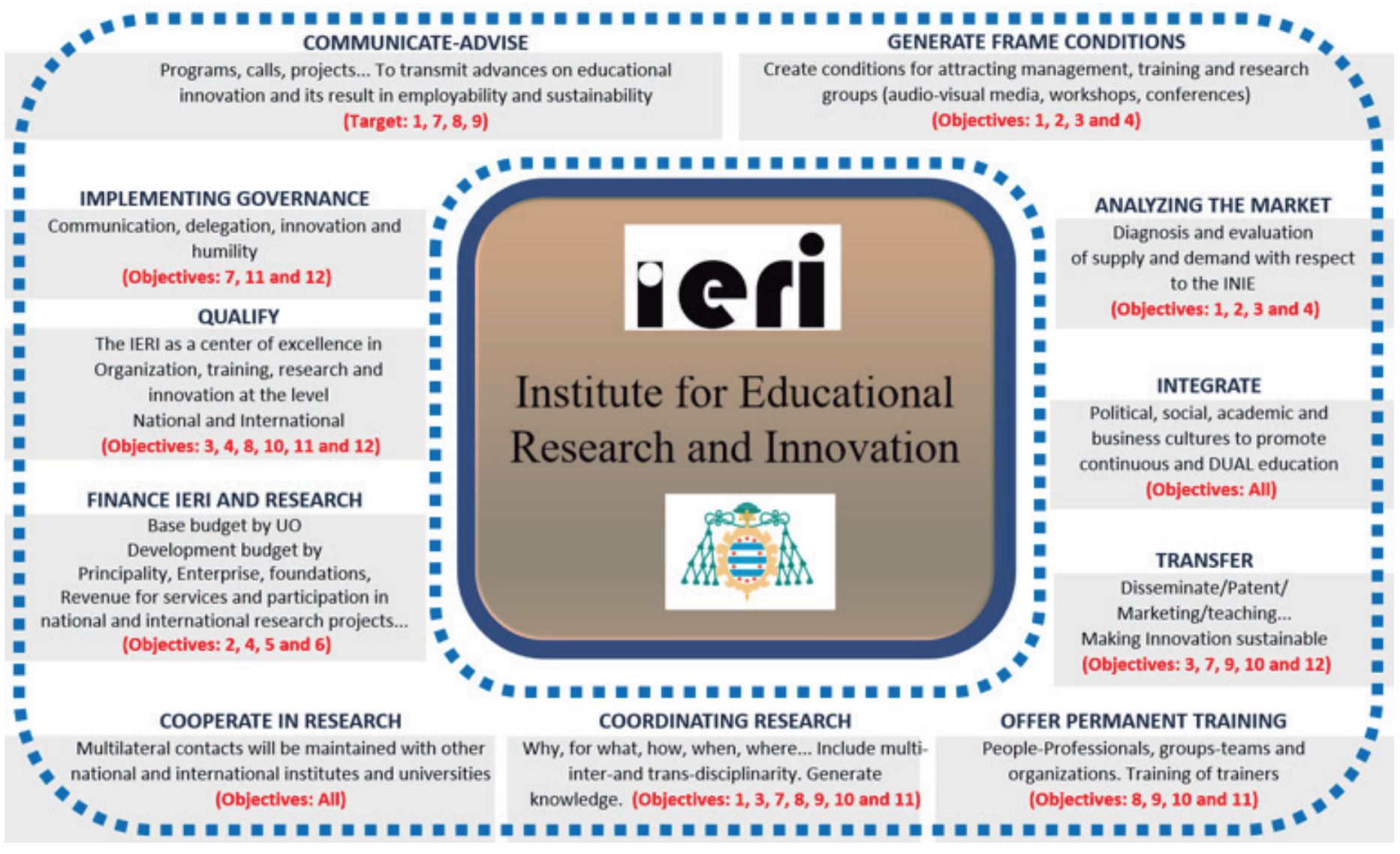

Chart 3. INIE's strategy and actions to promote Educational $R+D+i e$

The actions promoted from these references are generating added value to people, users, professionals and community), to methodologies, tools, protocols, technologies, systems and institutions.

The final objective is to generate Educational Innovation, although we are aware that this can occur in each of the actions, in a combined or global manner. Therefore, there is no linear cause-effect relationship between actions, but rather the process of change is understood as circular, systemic and timeless, where learning and teaching is considered as a continuum in which the stakeholders, namely academic, political, social, Labor and business, must participate. By way of example, we present the actions that we are currently promoting from the INIE, as they exemplify quite clearly what we mean, see figure 4 (ÁlvarezArregui, Rodríguez-Martín, Agudo Prado and Arreguit, 2017).
These actions are integrated into projects that can be coordinated by one or several entities and acquire their full potential when the propellers integrate the knowledge and experience of the cultures of the socio-political, academic, business and public administration environment. The projects that are deployed aim to provide answers, creative and coherent to the citizens through a multidisciplinary work that positively affects the personal, professional, institutional, local, regional, national and international development depending on the projection that you want to give Alvarez-Arregui, RodriguezMartin, Madrigal Maldonado, Grossi-Sampedro and Arreguit, 2017).

To close this point, we present an integrated vision of the ecosystem that we are developing, since it allows us to clarify the arguments that we have been making and where 


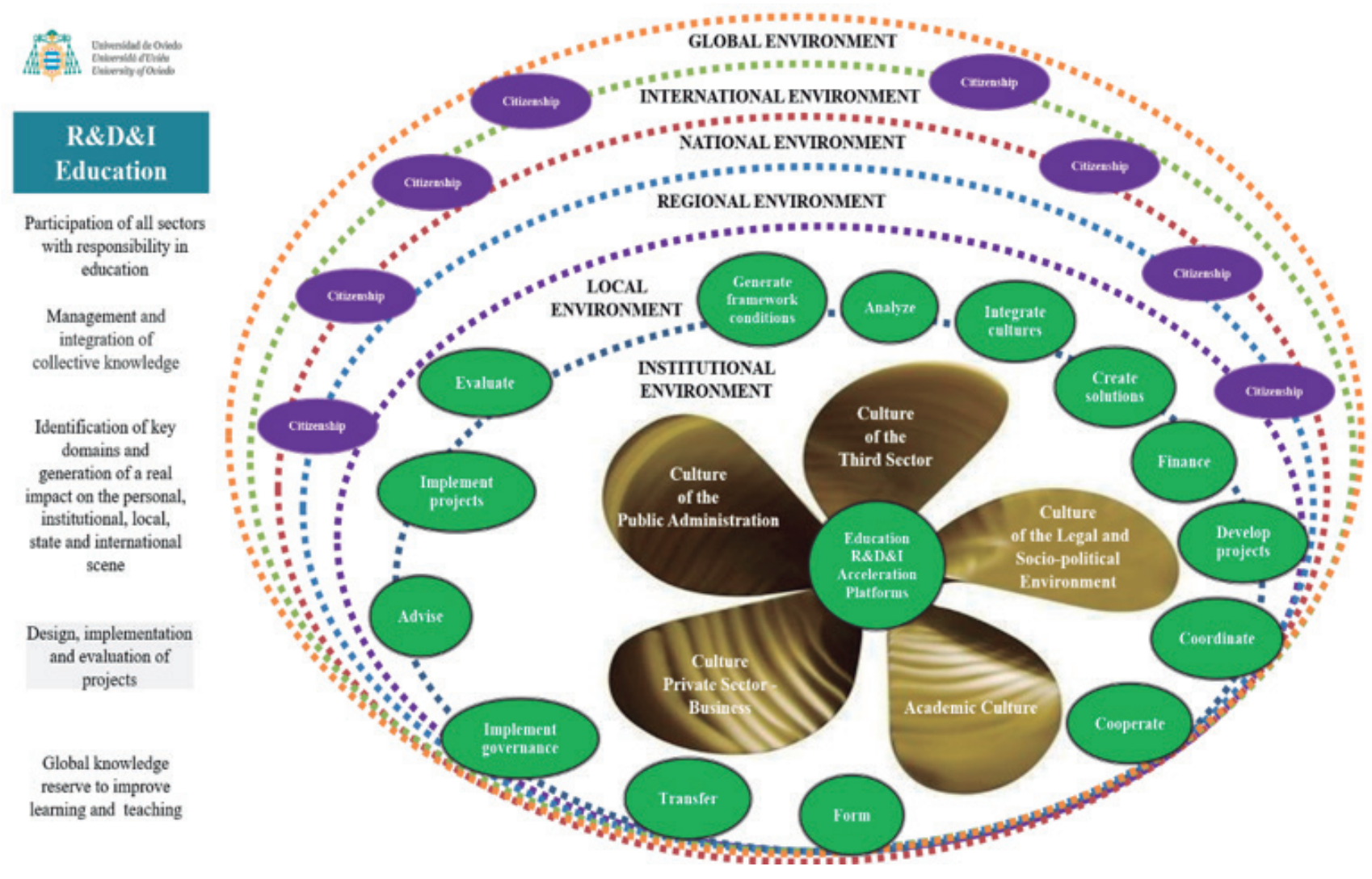

Chart 4. Glocal projection of the IERI understood as a platform for accelerating educational innovation

multiple contributions from theory, practice and experience are gathered in a synthetic way. As can be seen in the figure 5, the general structure is based on four basic reference components, an environment, a population, a system of relations and a technology.

Associated with each of them are some reference elements that are susceptible to multiple combinations and developments depending on the projects, objectives and priorities that have been established. In the central part appear the orientation of learning and teaching that is generated through the projects that are promoted, the values that guide them and the people, areas and geographical spaces on which they are projected.

By population component we understand the natural and legal persons that concur in a determined cultural and spatial environment, forming an ecosystem where the actors such as the students, the teachers, the families, the external advisors, the administration and services personnel, the local administration, the responsible and professionals of different political, social, educational and labour entities are elements to consider according to the project. The population has its own axioms (Hawley, 1982). Thus, we can speak of optimal or unadjusted levels depending on the number of participants, of a more or less adequate size depending on the degree of specialization required or of more or less appropriate spaces and times based on the technology or systems that one wishes to establish, among other issues.

The systems of interaction that are established in an ecosystem are fundamental to fulfilling its objectives and will determine the unique structure of the ecosystem. These relationships are presented in a static way when we refer to organizational charts or formal relationship systems and will be considered as dynamic when considering informal, ad hoc, research advisory training or innovation relationship systems. Obviously, the relationships will not be symbiotic (collaboration) in all cases, but there will be power systems that will be established based on the interests of the participants, and conflicts will emerge that will be addressed as another learning and teaching process. We also address in the same way the different levels of competence, ideological divergences, emotions, experience or dependence.

Ecosystems need energy to survive, so they need relevant and unique tools to capture and transform it. In this context, we think that Duncam's (1959: 689) arguments are correct when he refers to technology in human ecology "... not only to a complex of art and artefacts whose patterns are invented, disseminated and accumulated..., but to a set of techniques employed by a population to earn the sustenance of its environment and to facilitate the organization of the activity producing that sustenance. In the image you can see some common projects associated with technology in socio-educational environments although these will change based on the type of project, the population and the environment. In the latter, we distinguish between tangible structures (infrastructures, spaces, times, resources and materials), the intangible case of virtual spaces (interpersonal, administrative, academic, political, business...), the symbolic (associated with the climate and cultures generated in the organizations) and the political that are determined by the leadership systems that are established at different levels.

This approach shows the complexity of the environment, which entails interpreting it beyond a mere continent for the development of educational, curricular, training or research actions, since it becomes itself an object of study, learning, teaching and training. It is therefore essential to take this 


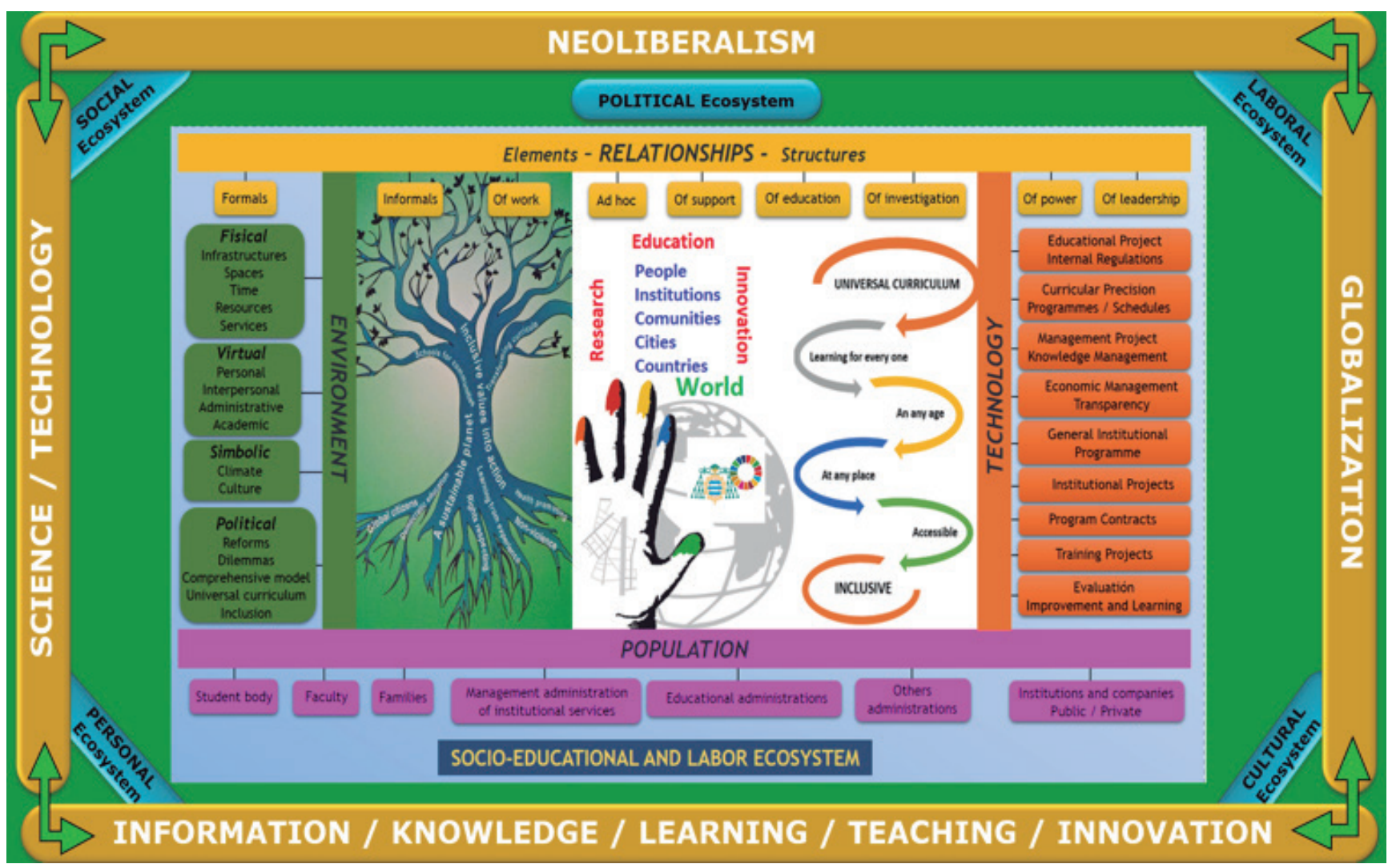

Chart 5. General vision of the INIE understood as an Ecosystem of Creative and Entrepreneurial Learning and Teaching (Álvarez-Arregui, 2017)

component into account when developing educational projects and actions, since it is a determining factor in promoting cultural change that can be conceptualized, analysed, reconstructed and improved.

The central part of the image contains the principles and values that guide the development of the projects in this model of educational innovation, thus integrating, among others, the principles of educating cities, the Index for Inclusion and the Objectives of the Agenda 2030 for Sustainable Development where universities play an essential role. The curriculum is designed considering the principles that guide the Universal Learning Design (ULD), will be multidisciplinary and will favour the learning of all people, at any age and in any place.

Generic and specific skills will be addressed, learned and taught through active blended-learning methodologies in order to promote an attitudinal and cultural change where the research for alternatives, the solution of problems, the use of new services, the implementation of products or the management of a methodology will be based on a systematic and systemic process of continuous training where participants will learn and teach to do through creative projects, learn and teach to innovate from transfer, learn and teach to learn from evaluation, training and research and learn and teach to be by sharing values and manifesting them in professional and social practice.

\section{Looking to the future: Lifelong Learning Ecosystems for a Sustainable and Responsible Learning Society}

Faced with a complex reality that is multidimensional and dynamic, simple, exclusive or generalizable solutions are no longer viable. The European Union is aware of this and since the Agenda 2030 for Sustainable Development has endorsed an international commitment and a timetable for tackling the social, economic and environmental challenges of globalisation, with the focus on people, the planet, prosperity and peace, under the motto of "leaving no one behind". From the University of Oviedo, in general, and from the Institute for Educational Research and Innovation (INIE), in particular, we consider that it is a great opportunity for improvement to mobilise all the administrations, at national, regional and local level, the citizens, the social actors, the companies, the universities, the research centres and the organisations of the civil society, around a shared vision that extends inside and outside our institution.

We are aware that the challenge is enormous, but it cannot be postponed and is urgent. This requires leading policies in the different areas of responsibility in the face of the multiple manifestations of poverty, inequalities, unemployment and degradation of our natural environment that are emerging, so all actors must be involved without exception. Therefore, the development and implementation of the ecosystem model of creative and entrepreneurial learning and teaching that we have presented is a clear commitment to the principles and actions that guide Agenda 2030. This reference represents an opportunity to reinforce the educational mission of the University in the full realization of human rights, peace, the responsible exercise of local and global citizenship, gender equality, sustainable development and health.

The contents of such education must include cognitive, procedural, attitudinal and value aspects as we set out in the principles that guide creative and entrepreneurial learning and teaching ecosystems. It is important, therefore, that the model responds to the commitments made in the framework of the ODS, preparing future cohorts of learners and teachers to live in a complex and interconnected world. Therefore, the curriculum 
should be open to incorporate content, actions and values that will provide people with the tools and skills necessary to understand the world and to manage it with a critical capacity guided by shared values. Within this framework we also consider, as does the International Labour Organization (ILO), the four pillars on which a Decent Work Agenda should be based - employment creation, social protection, rights at work and social dialogue. These references are fundamental elements for achieving the DWOs and essential for making the patterns of socio-educational and economic growth sustainable and inclusive. These and other issues have been taken into account in the design, development and implementation of our ecosystem and, more specifically, have been integrated into the Institute for Educational Research and Innovation of the University of Oviedo as well as other international ecosystems with which we collaborate through multiple agreements and arrangements. In our case we are convinced that from this model new views, visions, missions and strategies will be generated, in which citizens and actors from the socio-legal-political, academic and economic environments will participate more and more, and we will overcome the current limitations and situational problems and project ourselves into the future with greater guarantees of success.

In our opinion, it is no longer possible for the University to guarantee the maintenance of its current organisational structures, where teaching, research and transfer are governed by cultural indicators that are not aligned with the major emerging macro-trends. These institutions must review their staffing systems, because it is no longer acceptable for professionals with low management, teaching or research skills to continue to be incorporated, or to adopt a negative attitude towards continuous training or to continue to provide "basic" courses on an ongoing basis without changing the contents over the years. We also recognise that it is not viable to continue working in working environments where the intensification of working time has become a constant as a result of technological development and increased bureaucratisation, but this is no excuse to continue transmitting content encapsulated in disciplines, validated on the basis of corporate or individual criteria, without external control. The bureaucratic certification of competencies through periodic standardized evaluations that do not correspond between what is said to be done, what is said to be done, what is actually done and the results obtained, among other issues, is another important problem that will have to be resolved.

If education professionals, politicians, apprentices and entrepreneurs continue to be guided by these references, they will not be able and will not be able to commit themselves to the training, research and transfer that a learning and continuing education society requires. On the other hand, if a positive attitude towards change is adopted in the University, if the number of external professionals with temporary employment contracts from industry, public administrations or the service and technological sectors is increased, and if balances and collaboration are sought between all parties, we will be able to build the best possible curriculum, combining institutional knowledge guaranteed by academic freedom with the latest knowledge generated in the working, academic, social, legal and political environments.

In this new context of creative and enterprising learning and teaching, there will be a growing need for teachers to work transversally in their classes to enable students not only to learn and teach subjects, but also to know how to relate them in order to understand the complexity of the subjects being dealt with, which will necessarily lead them to pluri-disciplinarily and greater efficiency in transdisciplinary work. Therefore, we share those initiatives that go deeper into structural, functional and axiological pluralism, overcoming the limitations derived from bureaucratic, competitive and standardized models, in order to integrate the best of the public, private and voluntary sectors through an eco-training, multidisciplinary, entrepreneurial, inclusive and innovative approach.

Non-intervention is no longer an option because, if no commitments are made, disputes, contradictions, paradoxes, polemics and confrontations will continue to grow, as will gaps in literacy and in the ability to access resources for natural and legal persons in different parts of the planet. We are aware that the process of change is profound because it is cultural.

We would like to conclude by indicating that university excellence is and will be built with the active participation and commitment of all people and organizations, since from this platform it will be possible to create interactive links between the natural, social, business, political, technological and academic environments, which makes it necessary to generate creative, innovative, inclusive, interconnected, info-accessible and innovative learning ecosystems that will improve the quality of life of all people through educational $\mathrm{R}+\mathrm{D}+\mathrm{ie}$ and allow us to advance towards a human coexistence that guarantees a durable, desirable and sustainable future for current and future generations.

\section{Referencias / referents}

Alonso, L. E., Fernández, C. J. \& Nyssen, J. Mª (2009). El debate sobre las competencias. Una investigación cualitativa en torno a la educación superior y el mercado de trabajo en España. Madrid: ANECA.

Altbach, P. G. (2007). The Imperial Tongue: English as the Dominating Academic Language. Economic and Political Weekly, 8, 3608-3611.

Álvarez-Arregui, E. (2002). Acción directiva y cultura escolar. Influencia del liderazgo en el desarrollo institucional de los centros educativos. Oviedo: Universidad de Oviedo.

Álvarez-Arregui, E. (2008). El Espacio Europeo de Educación Superior (EEES) desde una perspectiva de cambio. Lecturas sobre el continente y el contenido de la docencia. I Congreso Internacional Nuevas Bases para el Diseño de los Planes de Estudio en el EEES y su Incidencia en el Sistema Educativo: Ecoformación, Transdisciplinariedad e Interculturalidad. Madrid: UNED.

Álvarez-Arregui, E. (2010). La Universidad desde una perspectiva de cambio: En busca de la excelencia. I Congresso Ibero-Brasileiro nos días 29 y 30 de abril en Elvas e 1 e 2 de Maio de 2010 en Mérida e Cáceres.

Álvarez-Arregui, E. (Coord.) (2017). Universidad, Investigación y Conocimiento: Avances y Retos. Oviedo: Servicio de Publicaciones de la Universidad de Oviedo.

Álvarez-Arregui, E. (Coord.) (2018). Universidad, Investigación y Conocimiento: comprensión e intervención en una sociedad compleja. Oviedo: Servicio de Publicaciones de la Universidad de Oviedo.

Álvarez-Arregui, E. (Coord.) (2019). Universidad, Investigación y Conocimiento: La Transversalidad como referente del desarrollo profesional y personal. Oviedo: Servicio de Publicaciones de la Universidad de Oviedo.

Álvarez-Arregui, E. y Rodríguez-Martín, A. (2015). Aprender a mirar las organizaciones desde una visión inclusiva. Avanzando desde la práctica hacia un modelo ecosistémico de formación y gestión. Oviedo: Editorial Ediuno.

Álvarez-Arregui, E.; Rodríguez-Martín, A.; Agudo Prado, S.; y Arreguit, X. (2017). Inclusive ecosystem model for the management of knowledge, training and Innovation: progress, difficulties and challenges. Aula Abierta, 46, 97-104. 
Álvarez-Arregui, E.; Rodríguez-Martín, A.; Madrigal Maldonado, R.; Grossi -Sampedro, B. G.; y Arreguit, X. (2017). Ecosystems of media training and competence. International assessment of its implementation in Higher Education. Comunicar, 51, XXV, 105-114.

Álvarez-Arregui, E.; Rodríguez-Martín, A.; San Fabián Maroto, J. L.; y Álvarez Fernández, M.ํㅡ. V. (2011). Una nueva encrucijada para las Políticas Públicas en Educación Superior. La universidad como inversión o la universidad como inversora. XXV Simposio Brasileiro de Política e Administraçao da Educaçao. Sao Paulo-Brasil: FEAE.

Álvarez-Arregui, E.; Rodríguez-Martín, A.; y Ribeiro Gonçalves, F. (2013). Los ecosistemas de formación blended learning en la práctica universitaria. Valoración de los estudiantes sobre su implementación. Revista Portuguesa de Educaçao, 26 (1), 143177.

Arreguit, X. (2016). Innovación Tecnológica y Gestión del Conocimiento. Documento empresarial interno policopiado. Lausanne: Innobridge.

Arreguit, X. (2018). Gestión del Conocimiento y Gobernanza de ecosistemas de base tecnológica. Documento empresarial interno policopiado. Lausanne: Innobridge.

Angulo, J. F. (2010). Educación y el curriculum en el espacio europeo ¿Internacionalizar o globalizar? En J. Gimeno Sacristán (Comp.) Saberes e incertidumbres sobre el currículum. Madrid: Morata.

Ash, M. G. (2006). Bachelor of What, Master of Whom? The Humboldt Myth and Historical Transformations of Higher Education in German-Speaking Europe and the US. European Journal of Education, 41(2), 245-267.

Barnett, R. (2005). Recapturing the universal in the university. Educational Philosophy and Theory, 37(6), 785-797.

Barr, R. \& Tagg, J. (1995). From teaching to learning: a new paradigm for undergraduate education. Change: The Magazine of Higher Learning, 27(6), 12-25.

Batory, A. \& Lindstrom, N. (2011). The Power of the Purse: Supranational Entrepreneurship, Financial Incentives, and European Higher Education Policy. Governance. An International Journal of Policy, Administration and Institutions, 24, 311-329.

Bolívar, A. (1999a). Cómo mejorar los centros educativos. Madrid. Editorial Síntesis.

Boni, A. \& Lozano, J. F. (2006). The generic competences: an opportunity for ethical learning in the European convergence in higher education. Higher Education, 545, 819-831.

Booth, T. \& Ainscow. M. (2015). Guía para la Educación Inclusiva. Desarrollando el aprendizaje y la participación en los centros escolares. Madrid. OEI/FUHEM.

Brodo, J. A. (2006). Today's Ecosystem of e-learning. Trainer Talk, Professional Society for Sales y Marketing Training, vol. 3, № 4, 2006.

Buckland, R. (2009). Private and Public Sector Models for Strategies in Universities. British Journal of Management, 20(4), 524536. (http://goo.gl/bPvxVt) (2016-11-25).

Carabaña, J. (2011). Competencias y Universidad, o un desajuste por mutual ignorancia. Bordón, 63(1), 15-31.

Castells, M. (1999). La era de la información. Fin de milenio. Vol. 3. Madrid: Alianza.

Chang, V., \& Lorna, U. (2008). Governance for e-learning ecosystem. In E. Chang, \& F. Hussain (Ed.). Second IEEE International Conference on Digital Ecosystems and Technologies, 340-345. Phitsanulok (Thailand): Institute of Electrical and Electronics Engineers.

COMISIÓN EUROPEA (2000). Por una Europa del conocimiento. Bruselas.
CONSEJO ECONÓMICO Y SOCIAL. Informe 03/2018 El futuro del trabajo. Madrid: Consejo Económico y Social.

De Miguel Díaz, M. (Dir.) (2006). Modalidades de enseñanza centradas en el desarrollo de competencias. Orientaciones para promover el cambio metodológico en el Espacio Europeo de Educación Superior. Oviedo. Universidad de Oviedo/MEC.

Delors, J. (1996). La educación encierra un tesoro. Informe de la UNESCO de la Comisión Internacional sobre la educación para el siglo XXI. Madrid: Santillana Ediciones UNESCO.

Dimitrov, V. (2001). Learning Ecology for Human and Machine Intelligence: A Soft Computing Approach. Studies in Fuzziness and Soft Computing, 81, 386-393. (http://goo.gl/TgXmvW) (2016-11-01).

Entwistle, N., \& Tait, H. (1990). Approaches to learning, evaluations of teaching, and preferences for contrasting academic environments. Higher Education, 19, 169-194. (http://goo.gl/ MKbfrs) (2016-08-09).

Escudero, J. M. (1999). El cambio en educación, las reformas y la renovación pedagógica. ESCUDERO, J. M. (ed.). Diseño, desarrollo e innovación. Currículum. Madrid. Síntesis. 76-96.

Fullan, M. (1991). The new meaning of Educational change. Nueva York/Londres. Teachers College. Press/Cassell. (Traducida al castellano por Octaedro-Barcelona en 2002 bajo el título Los nuevos significados del cambio en educación).

Gairín, J. (2003). El profesor universitario en el siglo XXI. MONEREO, C. \& POZO, J. I. (Eds.). La universidad ante la nueva cultura educativa. Enseñar y aprender para la autonomía. Barcelona. Síntesis. 119-139.

González, J. \& Wagenaar, R. (2003). Tuning Educational Structures in Europe. Informe final. Fase uno. Documento policopiado.

Goñi Zabala, J. (2005). El espacio europeo de educación superior, un reto para la universidad. Competencias, tareas y evaluación, los ejes del currículum universitario. Barcelona: Editorial Octaedro.

Gütl, C., \& Chang, V. (2009). Ecosystem-based Theorical Models for Learning in Environments of the 21st Century. International Journal of Emerging Technologies in Learning, 7, 1-11. (http://goo.gl/IhZOwg) (14-12-2015).

Hansen, D. T. (2008). Education Viewed Through a Cosmopolitan Prim, pp. 206-214. In R. D. Glass (ed.) Philosophy of Education Society. Urbana, Illinois: Philosophy of Education Society.

Hargreaves, A. (1998). Profesorado, cultura y posmodernidad. Cambian los tiempos, cambia el profesorado. Madrid. Morata. (1 $1^{\underline{a}}$ edición en castellano en 1996; original en inglés Changing Teachers, Changing Times. Teacher's work and Culture in the Postmodern Age, 1994. Londres. Cassell).

Hargreaves, A. y Fink, D. (2002). La sostenibilidad en el tiempo. Cuadernos de Pedagogía, 319, 16-20.

Ismail, J. (2001). The Design of an e-Learning System beyond the Hype. Internet and Higher Education, 4(3-4) 329-336. (http:// goo.gl/rqLPzA) (2017-12-17).

López Gómez, E. (2015). La tutoría en el EESS: propuesta, validación y valoración de un modelo integral. Tesis doctoral inédita.

Martín, E. (2003). Conclusiones. Un currículo para desarrollar la autonomía del estudiante. MONEREO, C. \& POZO, J. I. (Eds.), La universidad ante la nueva cultura educativa. Enseñar y aprender para la autonomía. Barcelona. Síntesis. 285-292.

Morín, E. (2009). Anotaciones para un nuevo Emilio: transmisión sistemática del conocimiento. Signo y Pensamiento, 56, $42-49$

Murga Menoyo, Maㅗ. \& Quicios García, Ma․ P. (coord.). La reforma de la universidad. Cambios exigidos para la nueva Europa. Madrid: Dykinson.

Pasias, G. \& Roussakis, Y. (2012). Políticas de Conocimiento Actuales en la Unión Europea: Diseño y Evaluación Críticos de 
la Calidad en una Europa del Conocimiento Medible. Revista Española de Educación Comparada, 20, 303-324.

Pérez-Bustamente Ilander, G. O., Álvarez-Arregui, E. y Rodríguez-Martín, A. (2013). New Teachers for New Competences. Oviedo. Universidad de Oviedo.

Perrenoud, P. (2004). Diez nuevas competencias para enseñar. Barcelona: Graó.

Popkewitz, Th. (1994). Sociología politica de las reformas educativas. El poder/saber en la enseñanza, la formación del profesorado y la investigación. Madrid. Morata-Fundación Paideia.

Power, M. (1999). The audit society: Rituals of verification. Oxford. Oxford University Press.

Rué Domingo, J. (2004). La convergencia europea entre decir e intentar hacer. Revista Interuniversitaria de Formación del Profesorado, 18(1), 39-59.

Ruiz Corbella, M. (2006). La universidad y el mercado del aprendizaje. Claves para comprender el concepto de competencia. MURGA MENOYO, A. y QUICIOS GARCÍA, Ma․ P. La reforma de la Universidad: cambios exigidos por la nueva Europa. Madrid: Dykinson. 93-114.

Santos Rego, M. A. (2014). Purpose of the University and Employability Challenges in Higher Education. Procedia-Social and Behavioral Sciences, 139, 16-25.

Schumpeter, J. (1943). Capitalism, Socialism and Democracy. London and New York: Routledge.

Scott, J. C. (2006). The mission of the university: medieval to postmodern transformations. The Journal of Higher Education, 77, 1-39

Senge, P. M. (1990). The Fifth Discipline. The Art and Practice of the Learning Organization. N. Y. Dobuleday. (Trad. esp. 1992, La quinta disciplina. El arte y la práctica de la organización abierta del aprendizaje. Barcelona: Granica).

Shrivastava, P. (1998). Knowledge Ecology: Knowledge Ecosystems for Business Education and Training. Lewisburg: Bucknell University Press.

Tennant, M., McMullen, D., \& Kaczynski, D. (2010). Teaching, learning and research in higher education: A critical approach. New York: Routledge.

Tonucci, F. (2009a). Ciudades a escala humana: la ciudad de los niños. Revista de Educación, número extraordinario, 147-168.

Uden, L., Wangsa, I.T., \& Damiani, E. (2007). The future of e-Learning: E-learning ecosystem. Digital EcoSystems and Technologies Conference, 7, 113 -117. https://doi.org/10.1109/ DEST.2007.371955.

Vélez, G. (2003). Enseñar estrategias de aprendizaje en la universidad. MONEREO, C. \& POZO, J. I. (Eds.), La universidad ante la nueva cultura educativa. Enseñar y aprender para la autonomía. Madrid. Síntesis. 215-229.

Villa, B. (2005). Sobre el lugar común: la Universidad humboldtiana puede ser correcta en teoría, pero no vale para la práctica: una breve introducción a tres textos de Humboldt sobre la Universidad. Logos: Anales del Seminario de Metafísica, 38, 273-281.

Weick, K. E. (1976). Educational Organizations as Loosely Coupled Systems. Administrative Science Quarterly, 21, 1-19.

Wilkinson, D. (2002). The Intersection of Learning Architecture and Instructional Design in e-Learning', ECI Conference on e-Technologies in Engineering Education: Learning Outcomes Providing Future Possibilities, 213-221. 
